\title{
INVARIANTS AND COINVARIANTS OF THE SYMMETRIC GROUP IN NONCOMMUTING VARIABLES
}

\author{
NANTEL BERGERON, CHRISTOPHE REUTENAUER, MERCEDES ROSAS, AND MIKE ZABROCKI
}

\begin{abstract}
We introduce a natural Hopf algebra structure on the space of noncommutative symmetric functions which was recently studied as a vector space by Rosas and Sagan 12. The bases for this algebra are indexed by set partitions. We show that there exist a natural inclusion of the Hopf algebra of noncommutative symmetric functions studied in 17 in this larger space. We also consider this algebra as a subspace of noncommutative polynomials and use it to understand the structure of the spaces of harmonics and coinvariants with respect to this collection of noncommutative polynomials.
\end{abstract}

\section{INTRODUCTION}

In the commutative world there are two constructions of the Hopf algebra of symmetric functions; the more classical one as the invariants of the symmetric group on the polynomial ring, the other is the commutative free algebra generated by one element in each degree (e.g. 9] vs. [13]). These two constructions lead to the same algebra.

In the noncommutative world, these perspectives lead to two very different algebras; a free algebra with one generator at each degree, NSym (see for instance [17] and references therein), and the algebra of noncommutative invariant polynomials, NCSym (studied in [2], [12, 18]). These algebras are clearly not isomorphic since the index set of a homogeneous basis of NSym is the set of compositions, with dimension $2^{n-1}$ at degree $n$, and NCSym is indexed by the set partitions, with dimension given by the Bell numbers for each graded component.

An obvious question that was first posed in the work of [12] was to understand the connection between the two algebras. In this paper we present an incredibly beautiful relationship between them. We answer this question by first introducing a natural Hopf algebra structure on the space of noncommutative polynomial invariants. The structure imposed by having both a product and a coproduct is much richer than just the algebra structure alone. This places so many conditions on a Hopf algebra embedding from NSym to NCSym that there is only one solution given a set of generators of Sym.

In the development of the proof that the embedding is injective, we compute the determinant of a combinatorial matrix indexed by compositions. It arises that the determinant is expressed as a product of the number of permutations with no global descents. This is a surprising fact since these numbers also happen to be the number of free generators/primitives of the Malvenuto-Reutenauer Hopf algebra of permutations (see [1, [5]). 
In section [5 digress and mention several other relationships between NCSym and NSym by examining some quotients and embeddings of the algebra structure. In particular, we show that, as a graded algebra, NCSym in two variables is isomorphic to NSym.

An interesting question that is natural to consider once one understands NCSym as the space of invariants is to try to understand the 'coinvariants.' An important classical theorem of Chevalley [4, and later extended to other finite reflection groups by Steinberg [16], says that the ring of polynomials is isomorphic as an $\mathfrak{S}_{n}$-module to the tensor product of its invariants times its coinvariants. We next ask ourselves if it is possible to obtain a version of Chevalley's theorem in the noncommutative setting.

The first step in answering this question is to determine what is meant by the coinvariants in noncommutative variables. In the commutative case there are two characterizations of this $\mathfrak{S}_{n}$-module. First, they can be defined as the solution space of the system of equations obtained by looking at symmetric functions without constant term as differential operators (e.g. $p_{2}(\partial) f=\nabla f=0$ ). The solution space is called the harmonics of the symmetric group. The coinvariants can also be defined as the quotient of $\mathbb{Q}[X] /\left\langle S y m_{n}^{+}\right\rangle$, where $\left\langle S y m_{n}^{+}\right\rangle$is the ideal generated by all symmetric functions without constant term. Indeed, in the commutative case these two definitions lead to isomorphic spaces.

In the noncommutative setting we have to be more careful. There are several possibilities for the meaning of a noncommutative derivative. First, we study study the harmonics of the symmetric group with regard to the Hausdorff derivative [11, the differential operator that acts on letters by $\partial_{a} b=\delta_{a, b}$, and that satisfies Leibniz rule $\partial_{a}(p q)=\left(\partial_{a} p\right) \cdot q+p \cdot\left(\partial_{a} q\right)$. We define the harmonics of the symmetric group in the noncommutative setting as the space of noncommutative polynomial solutions of the system of equations obtained by looking at symmetric functions without constant term as differential operators with regard to the Hausdorff derivative. We denote this space by $\mathrm{MHar}_{n}$.

In section 6] we give an elegant characterization of this space in terms of the free Lie algebra. We show that this space satisfies a mixed commutative/noncommutative version of Chevalley's theorem. More precisely, $\mathbb{Q}\left\langle X_{n}\right\rangle \simeq \operatorname{MHar}_{n} \otimes \operatorname{Sym}_{n}$ where $\operatorname{Sym}_{n}$ is the space of symmetric polynomials in $n$ variables.

In section 8, we look at the coinvariants of the symmetric group in noncommutative variables, defined as the left quotient

$$
\mathbb{Q}\left\langle X_{n}\right\rangle /\left\langle N C S y m_{n}^{+}\right\rangle
$$

where $\left\langle N C S y m_{n}^{+}\right\rangle$is the left ideal generated by the symmetric functions in $N C S y m_{n}$ without constant term. We obtain the Hilbert series of this space in terms of the number of Wolf's irreducible generators [18] which we present in a precise combinatorial manner in section 7.

In addition, we show that Chevalley's theorem holds in the noncommutative setting. More explicitely, we show that

$$
\mathbb{Q}\left\langle X_{n}\right\rangle \simeq \operatorname{NCSym}_{n} \otimes \mathbb{Q}\left\langle X_{n}\right\rangle /\left\langle N C \operatorname{Sym}_{n}^{+}\right\rangle .
$$

This is done by observing that the coinvariants of the symmetric group that we just described are isomorphic to the space of harmonic polynomials with respect to the twisted derivative defined by $d_{a}(b v)=\delta_{a, b} v$. 


\section{Combinatorics of Set Partitions}

A set partition $A$ of $m$ is a collection of nonempty subsets $A_{1}, A_{2}, \ldots, A_{k} \subseteq[m]=\{1,2, \ldots, m\}$ such that $A_{i} \cap A_{j}=\{\}$ for $i \neq j$ and $A_{1} \cup A_{2} \cup \cdots \cup A_{k}=[m]$. We will indicate that $A$ is a set partition of $m$ by the notation $A \vdash[m]$. The subsets $A_{i}$ are called the parts of the set partition and the number of nonempty parts is referred to as the length and will be denoted by $\ell(A)$.

There is a natural mapping from set partitions to integer partitions given by $\lambda(A)=$ $\left(\left|A_{1}\right|,\left|A_{2}\right|, \ldots,\left|A_{k}\right|\right)$, where we assume that the blocks of the set partition have been listed in weakly decreasing order of size. If $\lambda$ is a partition of $n$ (integer partition), we shall use $\ell(\lambda)$ to refer to the length (the number of parts) of the partition and $|\lambda|$ will be the size of the partition (the sum of the parts), while $n_{i}(\lambda)$ shall refer to the number of parts of the partition of size $i$. As a convention, lowercase Greek letters $\lambda, \mu$ and $\nu$ will be used to represent integer partitions while uppercase letters $A, B$ and $C$ will be used for set partitions.

When writing examples of set partitions we will use the notation that the sets of numbers are separated by the symbols, and the entire set partition is enclosed by $\{$ and $\}$. For example, $\{\{1,3,5\},\{2\},\{4\}\}$ will be represented in our notation by $\{135,2,4\}$. Although there is no order on the parts of a set partition, we will impose an implied order such that the parts are arranged by increasing value of the smallest element in the subset. This implied order will allow us to reference the $i^{t h}$ block of the set partition without ambiguity.

The number of set partitions is well known and given by the Bell numbers. These can be defined by the recurrence $B_{0}=1$ and $B_{n}=\sum_{i=0}^{n-1}\left(\begin{array}{c}n-1 \\ i\end{array}\right) B_{i}$. The next seven Bell numbers are $1,2,5,15,52,203,877$.

For a set $S=\left\{s_{1}, s_{2}, \ldots, s_{k}\right\}$ of integers $s_{i}$ and an integer $n$ we will use the notation $S+n$ to represent the set $\left\{s_{1}+n, s_{2}+n, \ldots, s_{k}+n\right\}$. For $A \vdash[m]$ and $B \vdash[r]$ set partitions with parts $A_{i}, 1 \leq i \leq \ell(A)$ and $B_{i}, 1 \leq i \leq \ell(B)$ respectively, we will set $A \mid B=\left\{A_{1}, A_{2}, \ldots, A_{\ell(A)}, B_{1}+m, B_{2}+m, \ldots, B_{\ell(B)}+m\right\}$, therefore $A \mid B \vdash[m+r]$ and that this operation is noncommutatative in the sense that, in general, $A|B \neq B| A$.

There is a natural lattice structure on the set partitions. We will define for $A, B \vdash[n]$ that $A \leq B$ if for each $A_{i} \in A$ there is a $B_{j} \in B$ such that $A_{i} \subseteq B_{j}$ (otherwise stated, that $A$ is finer than $B$ ). The set of set partitions of $[n]$ with this order forms a poset with rank function given by $n-k$ where $k$ the length of the set partition. This poset has minimal element $\{1,2, \cdots, n\}$ and maximal element $\{12 \cdots n\}$. The largest element smaller than both $A$ and $B$ will be denoted $A \wedge B=\left\{A_{i} \cap B_{j}: 1 \leq i \leq \ell(A), 1 \leq j \leq \ell(B)\right\}$ while the smallest element larger than $A$ and $B$ is denoted $A \vee B$.

Example 2.1. Let $A=\{138,24,5,67\}$ and $B=\{1,238,4567\}$. $A$ and $B$ are not comparable in the inclusion order on set partitions. We calculate that $A \wedge B=\{1,2,38,4,5,67\}$ and $A \vee B=\{12345678\}$.

When a collection of disjoint sets of positive integers is not a set partition because the union of the parts is not $[n]$ for some $n$, we may lower the values in the sets so that they keep their relative values so that the resulting collection is a set partition. This operation is referred to as the 'standardization' of a set of disjoint sets $A$ and the resulting set partition will be denoted $\operatorname{st}(A)$. 
Now for $A \vdash[m]$ and $S \subseteq\{1,2, \ldots, \ell(A)\}$ with $S=\left\{s_{1}, s_{2}, \ldots, s_{k}\right\}$, we define $A_{S}=$ $s t\left(\left\{A_{s_{1}}, A_{s_{2}}, \ldots, A_{s_{k}}\right\}\right)$ which will be a set partition of $\left|A_{s_{1}}\right|+\left|A_{s_{2}}\right|+\ldots+\left|A_{s_{k}}\right|$. By convention $A_{\{\}}$is the empty set partition.

Example 2.2. If $A=\{1368,2,4,579\}$, then

$$
\begin{array}{lll}
A_{\{1\}}=\{1234\} & A_{\{2\}}=\{1\} & A_{\{3\}}=\{1\} \\
A_{\{4\}}=\{123\} & A_{\{1,2\}}=\{1345,2\} & A_{\{1,3\}}=\{1245,3\} \\
A_{\{1,4\}}=\{1246,357\} & A_{\{2,3\}}=\{1,2\} & A_{\{2,4\}}=\{1,234\} \\
A_{\{3,4\}}=\{1,234\} & A_{\{1,2,3\}}=\{1356,2,4\} & A_{\{1,2,4\}}=\{1357,2,468\} \\
A_{\{1,3,4\}}=\{1257,3,468\} & A_{\{2,3,4\}}=\{1,2,345\} & A_{\{1,2,3,4\}}=\{1368,2,4,579\}
\end{array}
$$

\section{The Hopf Algebra of NONCOMMUtATIVE SYMmetric FUnCTIONS}

Consider for a fixed $n>0$ the space $\mathbb{Q}\left\langle X_{n}\right\rangle$ consisting of the linear span of monomials in noncommuting variables $X_{n}=\left\{x_{1}, x_{2}, \ldots, x_{n}\right\}$. There is a natural $\mathfrak{S}_{n}$ action on the basis elements defined by

$$
\sigma\left(x_{i_{1}} x_{i_{2}} \cdots x_{i_{k}}\right)=x_{\sigma\left(i_{1}\right)} x_{\sigma\left(i_{2}\right)} \cdots x_{\sigma\left(i_{k}\right)} .
$$

We can therefore consider $\mathbb{Q}\left\langle X_{n}\right\rangle$ as both an $\mathfrak{S}_{n}$ module and an algebra where the product of two monomials is given by the concatenation of the words.

Let $x_{i_{1}} x_{i_{2}} \cdots x_{i_{m}}$ be a monomial in the space $\mathbb{Q}\left\langle X_{n}\right\rangle$. We will say that the type of this monomial is the set partition $A \vdash[m]$ with the property that $i_{a}=i_{b}$ if and only if $a$ and $b$ are in the same block of the set partition. This set partition will be denoted as $\nabla\left(i_{1}, i_{2}, \ldots, i_{m}\right)=A$. Notice that the length of $\nabla\left(i_{1}, i_{2}, \ldots, i_{m}\right)$ will be equal to the number of different values which appear in $\left(i_{1}, i_{2}, \ldots, i_{m}\right)$.

The vector space $\mathrm{NCSym}_{n}$ will be defined as the linear span of the elements

$$
\mathbf{m}_{A}\left[X_{n}\right]=\sum_{\nabla\left(i_{1}, i_{2}, \ldots, i_{m}\right)=A} x_{i_{1}} x_{i_{2}} \cdots x_{i_{m}}
$$

for $A \vdash[m]$, where the sum is over all sequences with $1 \leq i_{j} \leq n$. For the empty set partition, we define by convention $\mathbf{m}_{\{\}}\left[X_{n}\right]=1$. If $\ell(A)>n$ we must have that $\mathbf{m}_{A}\left[X_{n}\right]=0$. Since for any permutation $\sigma \in S_{n}, \nabla\left(i_{1}, i_{2}, \ldots, i_{m}\right)=\nabla\left(\sigma\left(i_{1}\right), \sigma\left(i_{2}\right), \ldots, \sigma\left(i_{m}\right)\right)$, we also know $\sigma \mathbf{m}_{A}\left[X_{n}\right]=\mathbf{m}_{A}\left[X_{n}\right]$.

Example 3.1. We list below the monomial NCSFs corresponding to set partitions of size 3 in a polynomial algebra with 4 variables.

$$
\begin{array}{rc}
\mathbf{m}_{\{123\}}\left[X_{4}\right] & =x_{1} x_{1} x_{1}+x_{2} x_{2} x_{2}+x_{3} x_{3} x_{3}+x_{4} x_{4} x_{4} . \\
\mathbf{m}_{\{12,3\}}\left[X_{4}\right] & =x_{1} x_{1} x_{2}+x_{1} x_{1} x_{3}+x_{1} x_{1} x_{4}+x_{2} x_{2} x_{1}+x_{2} x_{2} x_{3}+x_{2} x_{2} x_{4}+ \\
\mathbf{m}_{\{13,2\}}\left[X_{4}\right] & x_{3} x_{3} x_{1}+x_{3} x_{3} x_{2}+x_{3} x_{3} x_{4}+x_{4} x_{4} x_{1}+x_{4}^{2} x_{2}+x_{4}^{2} x_{3} . \\
& x_{1} x_{2} x_{1}+x_{1} x_{3} x_{1}+x_{1} x_{4} x_{1}+x_{2} x_{1} x_{2} \cdot+x_{2} x_{3} x_{2}+x_{2} x_{4} x_{2}+ \\
\mathbf{m}_{\{23,1\}}\left[X_{4}\right] & x_{3} x_{1} x_{3}+x_{3} x_{2} x_{3}+x_{3} x_{4} x_{3}+x_{4} x_{1} x_{4}+x_{4} x_{2} x_{4}+x_{4} x_{3} x_{4} . \\
& x_{2} x_{1} x_{1}+x_{3} x_{1} x_{1}+x_{4} x_{1} x_{1}+x_{1} x_{2} x_{2} \cdot+x_{3} x_{2} x_{2}+x_{4} x_{2} x_{2}+ \\
\mathbf{m}_{\{1,2,3\}}\left[X_{4}\right]=\sum_{\sigma \in S_{4}} x_{\sigma(1)} x_{\sigma(2)} x_{\sigma(3)} .
\end{array}
$$


Now let $N_{\text {CSym }}$ be the space of polynomials of $\mathbb{Q}\left\langle X_{n}\right\rangle$ which are invariant under the action of $\mathfrak{S}_{n}$. For any element $f \in N C S y m_{n}$, if $\nabla\left(i_{1}, i_{2}, \ldots, i_{k}\right)=\nabla\left(j_{1}, j_{2}, \ldots, j_{k}\right)$ then the coefficient of $x_{i_{1}} x_{i_{2}} \cdots x_{i_{m}}$ in $f$ is equal to the coefficient of $x_{j_{1}} x_{j_{2}} \cdots x_{j_{k}}$ in $f$. We therefore conclude that $\left\{\mathbf{m}_{A}\left[X_{n}\right]\right\}_{\ell(A) \leq n}$ is a basis for $N C S y m_{n}$. In addition $N C$ Sym $_{n}$ has a ring structure where the product in this ring is defined as the natural extension of the ring structure on $\mathbb{Q}\left\langle X_{n}\right\rangle$.

Our motivation for the following definitions is to extend this algebra to a Hopf algebra. Define the vector space $N C S y m^{n}=\mathcal{L}\left\{\mathbf{m}_{A}\right\}_{A \vdash[n]}$ where here we have used $\mathbf{m}_{A}$ as a symbol representing a basis element for $A$ a set partition. $N C S y m=\bigoplus_{n>0} N C S$ Sm $^{n}$ is now the space of noncommutative symmetric functions (as opposed to the space of noncommutative symmetric polynomials). The degree of a basis element $\mathbf{m}_{A}$ is given by $|A|$. This graded vector space is endowed with a product map $\mu: N_{\text {CSym }}^{n} \otimes$ NCSym $^{m} \longrightarrow$ NCSym $^{m+n}$ which is defined on the basis elements $\mathbf{m}_{A} \otimes \mathbf{m}_{B}$ by

$$
\mu\left(\mathbf{m}_{A} \otimes \mathbf{m}_{B}\right):=\sum_{C \vdash[m+n]} \mathbf{m}_{C}
$$

where the sum is over all set partitions $C$ of $m+n$ such that $C \wedge(\{1 \ldots n\} \mid\{1 \ldots m\})=(A \mid B)$.

This definition is chosen to agree with the product map defined on $\mathbf{m}_{A}\left[X_{n}\right]$ since we have the following proposition.

Proposition 3.2. Let $A \vdash[r]$ and $B \vdash[m]$, we have

$$
\mathbf{m}_{A}\left[X_{n}\right] \mathbf{m}_{B}\left[X_{n}\right]=\sum_{C} \mathbf{m}_{C}\left[X_{n}\right]
$$

where the sum is over all set partitions $C$ of $r+m$ such that $C \wedge(\{1 \ldots r\} \mid\{1 \ldots m\})=(A \mid B)$ with $\ell(C) \leq n$.

Proof. The coefficient of any monomial $x_{i_{1}} x_{i_{2}} \cdots x_{i_{m+r}}$ in the expression $\mathbf{m}_{A}\left[X_{n}\right] \mathbf{m}_{B}\left[X_{n}\right]$ as a product in $\mathbb{Q}\left\langle X_{n}\right\rangle$ will have the value either 1 or 0 . As we are working in $\mathbb{Q}\left\langle X_{n}\right\rangle$ we must have that $\ell\left(\nabla\left(i_{1}, \ldots, i_{r+m}\right)\right) \leq n$. We note that the coefficient will be 1 if and only if $\nabla\left(i_{1}, \ldots, i_{r}\right)=A$ and $\nabla\left(i_{r+1}, \ldots, i_{r+m}\right)=B$. This will hold if and only if $\nabla\left(i_{1}, \ldots, i_{r+m}\right) \cap$ $\{1, \ldots, r\}=A$ and $\nabla\left(i_{1}, \ldots, i_{r+m}\right) \cap\{r+1, \ldots, r+m\}=B+r$. This is exactly equivalent to the condition that $\nabla\left(i_{1}, \ldots i_{r+m}\right) \wedge(\{1 \ldots r\} \mid\{1 \ldots m\})=A \mid B$.

We can conclude that for any $n$, the map $\phi_{n}: N C S y m \rightarrow N C S y m_{n}$ is a surjective algebra homomorphism where $\phi_{n}$ is defined as the linear function whose action on the basis is given by $\phi_{n}\left(\mathbf{m}_{A}\right)=\mathbf{m}_{A}\left[X_{n}\right]$ for $\ell(A) \leq n$ and $\phi_{n}\left(\mathbf{m}_{A}\right)=0$ otherwise. We state this precisely in the following corollary.

Corollary 3.3. The map $\phi_{n}$ is an algebra morphism. That is,

$$
\phi_{n}\left(\mu\left(\mathbf{m}_{A} \otimes \mathbf{m}_{B}\right)\right)=\phi_{n}\left(\mathbf{m}_{A}\right) \phi_{n}\left(\mathbf{m}_{B}\right)
$$

Even though it is defined as an abstract algebra, NCSym can be realized as the formal series of bounded degree in an infinite number of variables which are invariant under all permutations of the indices. The map $\phi_{n}$ is the specialization of this algebra so that the variables $x_{n+1}=x_{n+2}=x_{n+3}=\cdots=0$. In fact we have,

$$
\phi_{n}(F)=0 \text { for all } n \geq 1 \text { if and only if } F=0 \text {. }
$$


The algebra NCSym was originally considered by Wolf [18] in extending the fundamental theorem of symmetric functions to this algebra and later by Bergman and Cohn [2]. More recently Rosas and Sagan [12] considered this space to define natural bases which generalize the bases of the symmetric functions. Our point of departure is to consider NCSym as a Hopf algebra so that we may examine it from another perspective.

To this end we define a coproduct map $\Delta: N C$ Sym $^{n} \longrightarrow \bigoplus_{k=0}^{n} \operatorname{NCSym}^{k} \otimes$ NCSym $^{n-k}$ as

$$
\Delta\left(\mathbf{m}_{A}\right)=\sum_{S \subseteq[\ell(A)]} \mathbf{m}_{A_{S}} \otimes \mathbf{m}_{A_{S^{c}}}
$$

where $S^{c}=[\ell(A)] \backslash S$.

Assume that the $X_{n}$ and $Y_{n}$ are two sets of variables which each set is noncommutative but we have the relations $x_{i} y_{j}=y_{j} x_{i}$. Let $\phi_{n}^{X}\left(\mathbf{m}_{A}\right)=\mathbf{m}_{A}\left[X_{n}\right]$ and $\phi_{n}^{Y}\left(\mathbf{m}_{A}\right)=\mathbf{m}_{A}\left[Y_{n}\right]$, as before.

Proposition 3.4. For $F \in N C S y m$, we have

$$
\psi \circ\left(\phi_{n}^{X} \otimes \phi_{n}^{Y}\right) \circ \Delta(F)=F\left[X_{n}, Y_{n}\right]
$$

where $F\left[X_{n}, Y_{n}\right]$ represents the noncommutative symmetric polynomial in $2 n$ variables with the additional relations mentioned above and $\psi\left(f\left[X_{n}, Y_{n}\right] \otimes g\left[X_{n}, Y_{n}\right]\right)=f\left[X_{n}, Y_{n}\right] g\left[X_{n}, Y_{n}\right]$.

Proof. It suffices to prove this relation for the $\mathbf{m}_{A}$ basis. We know then that

$$
\psi \circ\left(\phi_{n}^{X} \otimes \phi_{n}^{Y}\right) \circ \Delta\left(\mathbf{m}_{A}\right)=\sum_{S \subseteq[\ell(A)]} \mathbf{m}_{A_{S}}\left[X_{n}\right] \mathbf{m}_{A_{S^{c}}}\left[Y_{n}\right] .
$$

Now in addition

$$
\mathbf{m}_{A}\left[X_{n}, Y_{n}\right]=\sum_{\nabla\left(i_{1}, i_{2}, \ldots, i_{r}\right)=A} x_{i_{1}} x_{i_{2}} \cdots x_{i_{r}}
$$

where the sum is over the sequences with $1 \leq i_{k} \leq 2 n$ and we are identifying $x_{i+n}=y_{i}$ for $1 \leq i \leq n$. Now for each part of $A, A_{i}=\left\{k_{1}, k_{2}, \ldots, k_{\left|A_{i}\right|}\right\}$, has $i_{k_{1}}=i_{k_{2}}=\cdots=i_{k_{\left|A_{i}\right|}}$. For a fixed $S \subseteq[\ell(A)]$, consider only the terms with the property that if $i \in S$ and $i_{k} \in A_{i}$ then $1 \leq i_{k} \leq n$ and if $i \notin S$ then all $i_{k} \in A_{i}$ will have $n+1 \leq i_{k} \leq 2 n$ (that is $x_{i_{k}}=y_{i_{k}-n}$ ). If we restrict the sum to these sequences, then we have

$$
\sum_{\substack{\nabla\left(i_{1}, i_{2}, \ldots, i_{r}\right)=A \\ i_{k}<n+1}}^{\Longleftrightarrow i_{k} \in A_{i}, i \in S} \underset{i_{1}}{ } x_{i_{2}} \cdots x_{i_{r}}=\mathbf{m}_{A_{S}}\left[X_{n}\right] \mathbf{m}_{A_{S^{c}}}\left[Y_{n}\right] .
$$

This implies

$$
\begin{aligned}
\mathbf{m}_{A}\left[X_{n}, Y_{n}\right] & =\sum_{S \subseteq[\ell(A)]} \sum_{\substack{\nabla\left(i_{1}, i_{2}, \ldots, i_{r}\right)=A \\
i_{k}<n+1}} x_{i_{1}} x_{i_{2}} \cdots x_{i_{r}} \\
& =\sum_{S \subseteq[\ell(A)]} \mathbf{m}_{A_{S}}\left[X_{n}\right] \mathbf{m}_{A_{S^{c}}}\left[Y_{n}\right]
\end{aligned}
$$

and this is equal to (7). 
In order to have a Hopf algebra we need, in addition, that the coproduct map is an algebra morphism in the following sense.

Proposition 3.5. Let $\tau(F \otimes G)=G \otimes F$ for $F, G \in N C$ Sym, then

$$
\Delta \circ \mu=(\mu \otimes \mu) \circ(i d \otimes \tau \otimes i d) \circ(\Delta \otimes \Delta) .
$$

Proof. We will use the previous results and (15) to derive this identity. First we note that for $F, G \in N C S y m$, and for any $n$ we have by Proposition 3.2 and Proposition 3.4 .

$$
\psi \circ\left(\phi_{n}^{X} \otimes \phi_{n}^{Y}\right) \circ \Delta \circ \mu(F \otimes G)=F\left[X_{n}, Y_{n}\right] G\left[X_{n}, Y_{n}\right]
$$

The fact that the $X_{n}$ and $Y_{n}$ variables commute implies that $\psi \circ \tau \circ\left(\phi_{n}^{X} \otimes \phi_{n}^{Y}\right)=\psi \circ\left(\phi_{n}^{X} \otimes \phi_{n}^{Y}\right)$.

Therefore, since

$$
\begin{aligned}
\psi \circ\left(\phi_{n}^{X}\right. & \left.\otimes \phi_{n}^{Y}\right) \circ(\mu \otimes \mu) \circ(i d \otimes \tau \otimes i d) \circ(\Delta \otimes \Delta)\left(\mathbf{m}_{A} \otimes \mathbf{m}_{B}\right) \\
& =\sum_{S \subseteq[\ell(A)]} \sum_{T \subseteq[\ell(B)]} \mathbf{m}_{A_{S}}\left[X_{n}\right] \mathbf{m}_{B_{T}}\left[X_{n}\right] \mathbf{m}_{A_{S^{c}}}\left[Y_{n}\right] \mathbf{m}_{B_{T^{c}}}\left[Y_{n}\right] \\
& =\sum_{S \subseteq[\ell(A)]} \mathbf{m}_{A_{S}}\left[X_{n}\right] \mathbf{m}_{A_{S^{c}}}\left[Y_{n}\right] \sum_{T \subseteq[\ell(B)]} \mathbf{m}_{B_{T}}\left[X_{n}\right] \mathbf{m}_{B_{T^{c}}}\left[Y_{n}\right] \\
& =\mathbf{m}_{A}\left[X_{n}, Y_{n}\right] \mathbf{m}_{B}\left[X_{n}, Y_{n}\right] .
\end{aligned}
$$

Now, since it suffices to prove the relation for the $\mathbf{m}_{A}$ basis and by Proposition 3.4

$$
\psi \circ\left(\phi_{n}^{X} \otimes \phi_{n}^{Y}\right) \circ \Delta \circ \mu=\psi \circ\left(\phi_{n}^{X} \otimes \phi_{n}^{Y}\right) \circ(\mu \otimes \mu) \circ(i d \otimes \tau \otimes i d) \circ(\Delta \otimes \Delta)
$$

holds for any $n$, we must have that equation (8) holds on NCSym.

\section{Hopf Algebras}

One of the main reasons for looking at this space as a Hopf algebra is that we are able to put it in context with other well known Hopf algebras. To this end, we introduce the space of symmetric functions Sym and another algebra referred to as the noncommutative symmetric functions NSym.

For each graded bialgebra $\mathcal{H}$, we will have an implicit unit map $u^{\mathcal{H}}$ sending the 1 in the field to the degree 0 basis element (also denoted by 1 ) and the counit $\varepsilon^{\mathcal{H}}$ which sends all terms of degree greater than 0 to 0 and the 1 of the algebra to the 1 of our base field (in the following algebras will always be $\mathbb{Q}$ ).

In each of the following bialgebras, the product $\mu^{\mathcal{H}}$ and coproduct $\Delta^{\mathcal{H}}$ respect the grading in the sense that $\mu^{\mathcal{H}}: \mathcal{H}^{n} \otimes \mathcal{H}^{m} \rightarrow \mathcal{H}^{n+m}$ and $\Delta^{\mathcal{H}}: \mathcal{H}^{n} \rightarrow \bigoplus_{k=0}^{n} \mathcal{H}^{k} \otimes \mathcal{H}^{n-k}$ where we will indicate the vector spaces $\mathcal{H}^{n}$ as the homogeneous components of the algebra of degree $n$. It is a well known result that every graded bialgebra where the degree 0 component has dimension 1 is a Hopf algebra (i.e., is a connected Hopf algebra) 15.

We will not give the antipode explicitly on the algebras but it is defined uniquely for any graded, connected Hopf algebra by the defining relation:

$$
\mu^{\mathcal{H}} \circ\left(i d \otimes S^{\mathcal{H}}\right) \circ \Delta^{\mathcal{H}}=u^{\mathcal{H}} \circ \varepsilon^{\mathcal{H}} \text {. }
$$


To compute the action of the antipode on an element $f$ of homogeneous degree greater than 0 , we write $\Delta^{\mathcal{H}}(f)=1 \otimes f+\sum_{i} g_{i}^{\prime} \otimes g_{i}^{\prime \prime}$. It then follows that

$$
0=\mu^{\mathcal{H}} \circ\left(i d \otimes S^{\mathcal{H}}\right) \circ \Delta^{\mathcal{H}}(f)=S^{\mathcal{H}}(f)+\sum_{i} \mu^{\mathcal{H}}\left(g_{i}^{\prime} \otimes S^{\mathcal{H}}\left(g_{i}^{\prime \prime}\right)\right)
$$

which can be used to solve for $S^{\mathcal{H}}(f)$ while the $S^{\mathcal{H}}\left(g_{i}^{\prime \prime}\right)$ will be of smaller degree and can be computed recursively.

From this discussion and Proposition 3.5 we can conclude

Theorem 4.1. NCSym is a Hopf algebra.

4.1. Symmetric functions. We will define the algebra of symmetric functions Sym as the free commutative algebra generated by elements $h_{k}$ for $k \geq 1$. The product on this algebra is the standard commutative product with a grading defined by $\operatorname{deg}\left(h_{k}\right)=k$ and following convention we will denote $S y m^{n}=\mathcal{L}\left\{h_{\lambda}\right\}_{\lambda \vdash n}$ with $h_{\lambda}:=h_{\lambda_{1}} h_{\lambda_{2}} \cdots h_{\lambda_{\ell(\lambda)}}$ and set Sym $=\bigoplus_{n \geq 0}$ Sym $^{n}=\mathbb{Q}\left[h_{1}, h_{2}, h_{3}, \ldots\right]$.

We may define the graded dual algebra $S y m^{*}$ by defining the graded component of degree $n$ is the vector space defined $\left(S y m^{*}\right)^{n}=\mathcal{L}\left\{m_{\lambda}\right\}_{\lambda \vdash n}$ and space is $S y m^{*}=\bigoplus_{n>0}\left(S y m^{*}\right)^{n}$ where the basis $m_{\lambda}$ is dual to $h_{\lambda}$ in the dual pairing. It arises that $S y m^{*} \simeq S y m$. In fact,

$$
h_{n}=\sum_{\lambda \vdash n} m_{\lambda} .
$$

The product and coproduct on the $m_{\lambda}$ basis can be determined from the product and coproduct on the $h$-basis. It develops that,

$$
m_{\lambda} m_{\mu}=\sum_{\nu} r_{\lambda \mu}^{\nu} m_{\nu}
$$

where the coefficients $r_{\lambda \mu}^{\nu}$ are the number of pairs of vectors $(\alpha, \beta)$ such that $\alpha \sim \lambda, \beta \sim \mu$ such that $\alpha_{i}+\beta_{i}=\nu_{i}$ for all $i$ and $\alpha \sim \lambda$ means that the sequence of values of $\alpha$ rearranges to the partition $\lambda$.

The coproduct is given by the formulas

$$
\Delta^{\text {Sym }}\left(h_{n}\right)=\sum_{k=0}^{n} h_{k} \otimes h_{n-k}
$$

and

$$
\Delta^{S y m^{*}}\left(m_{\lambda}\right)=\sum_{\mu \uplus \nu=\lambda} m_{\mu} \otimes m_{\nu}
$$

where we have denoted in the sum by $\mu \uplus \nu=\lambda$ that $\mu$ and $\nu$ are partitions satisfying $n_{i}(\nu)+n_{i}(\mu)=n_{i}(\lambda)$ for all $i \geq 1$.

4.2. The other Hopf algebra of noncommutative symmetric functions. There exists a noncommutative algebra which can be seen as an analogue to Sym (while NCSym = $\bigoplus_{n \geq 0} \mathcal{L}\left\{\mathbf{m}_{A}\right\}_{A \vdash[n]}$ is more clearly an analogue of $S y m^{*}=\bigoplus_{n \geq 0} \mathcal{L}\left\{m_{\lambda}\right\}_{\lambda \vdash n}$ ), see for example [17. NSym is defined as the noncommutative polynomial ring generated freely by elements $\mathbf{h}_{k}$ for $k \geq 1$ where $\operatorname{deg}\left(\mathbf{h}_{k}\right)=k$. For a composition $\alpha$ of $n$ (denoted $\alpha \models n$ ) we 
set $\mathbf{h}_{\alpha}:=\mathbf{h}_{\alpha_{1}} \mathbf{h}_{\alpha_{2}} \cdots \mathbf{h}_{\alpha_{\ell(\alpha)}}$ and $N \operatorname{Sym}^{n}=\mathcal{L}\left\{\mathbf{h}_{\alpha}\right\}_{\alpha \models n}$. The graded algebra is then defined as

$$
N S y m=\bigoplus_{n \geq 0} N S y m^{n}=\mathbb{Q}\left\langle\mathbf{h}_{1}, \mathbf{h}_{2}, \mathbf{h}_{3}, \ldots\right\rangle .
$$

The compositions of $n$ are in bijection with the subsets of $\{1,2, \ldots, n-1\}$ by the correspondence $D(\alpha)=\left\{\alpha_{1}, \alpha_{1}+\alpha_{2}, \ldots, \alpha_{1}+\alpha_{2}+\cdots+\alpha_{\ell(\alpha)-1}\right\}$ (the descent set of the composition $\alpha)$ and hence $\operatorname{dim}\left(N \operatorname{Sym}^{n}\right)=2^{n-1}$.

The product on $N$ Sym is defined so that $\mu^{N S y m}: N S y m^{n} \otimes N S y m^{m} \rightarrow N S y m^{n+m}$ as the free noncommutative product $\mu^{N S y m}\left(\mathbf{h}_{\alpha} \otimes \mathbf{h}_{\beta}\right)=\mathbf{h}_{\alpha} \mathbf{h}_{\beta}$.

The coproduct is given by the following formula and the fact that $\Delta^{N S y m}$ is an algebra homomorphism.

$$
\Delta^{N S y m}\left(\mathbf{h}_{n}\right)=\sum_{k=0}^{n} \mathbf{h}_{k} \otimes \mathbf{h}_{n-k}
$$

There is a significant difference between the dimensions of the two Hopf algebras of noncommutative symmetric functions. The dimension of $N C S y m^{n}$ is the number of set partitions of $[n]$, and for $n>2$ this is larger than the dimension of $N S y m^{n}$ which is the number of compositions of $n$ or $\operatorname{dim}\left(N\right.$ Sym $\left.^{n}\right)=2^{n-1}$.

4.3. Relations between Sym, NSym, and NCSym. The symbol $\chi$ will represent the 'forgetful' map which sends elements of a noncommutative algebra to the commutative counterpart (the map which 'forgets' that expressions are noncommutative). In our case we will begin by considering two such maps. The first of which is $\chi: N S y m \rightarrow$ Sym given by the linear homomorphism $\chi\left(\mathbf{h}_{\alpha}\right)=h_{\alpha_{1}} h_{\alpha_{2}} \cdots h_{\alpha_{\ell(\alpha)}}$.

Proposition 4.2. The linear map $\chi: N$ Sym $\rightarrow$ Sym where $\chi\left(\mathbf{h}_{\alpha}\right)=h_{\alpha}$ is a Hopf morphism.

Proof. This is easy to check on the $\mathbf{h}_{\alpha}$ basis since $\chi\left(\mathbf{h}_{\alpha} \mathbf{h}_{\beta}\right)=h_{\alpha} h_{\beta}$ and

$$
(\chi \otimes \chi) \circ \Delta^{N \operatorname{Sym}}\left(\mathbf{h}_{n}\right)=\sum_{k=0}^{n} h_{k} \otimes h_{n-k}=\Delta^{S y m} \circ \chi\left(\mathbf{h}_{n}\right) .
$$

Since both $\chi$ and $\Delta$ are algebra homomorphisms, this relation will hold as well on basis elements $\mathbf{h}_{\alpha}$.

In addition we will use the same symbol $\chi$ to represent the map $\chi: N C S y m \rightarrow$ Sym $\cong$ Sym* ${ }^{*}$ given by the linear homomorphism $\chi\left(\mathbf{m}_{A}\right)=\lambda(A)^{!} m_{\lambda(A)}$ where we denote $\lambda^{!}=$ $\left(\prod_{i \geq 1} n_{i}(\lambda) !\right)$. By contrast, we will use $\lambda !=\lambda_{1} ! \lambda_{2} ! \cdots \lambda_{\ell(\lambda)}$ ! (these conventions use the notation introduced in section 2 and are consistent with the notation of 12$]$ ). This map is inspired from the expression $\mathbf{m}_{A}\left[X_{n}\right]$ since if the variables were allowed to commute then the expression is equal to $\lambda(A)^{!} m_{\lambda(A)}\left[X_{n}\right]$ where for a partition $\lambda, m_{\lambda}\left[X_{n}\right]=\sum_{\alpha \sim \lambda} x^{\alpha}$ is the monomial symmetric polynomial.

Proposition 4.3. The linear map $\chi: N C S y m \rightarrow$ Sym where $\chi\left(\mathbf{m}_{A}\right)=\lambda(A)^{!} m_{\lambda(A)}$ is a Hopf morphism. 
Proof. As we remarked above, $\chi: N C S y m \rightarrow$ Sym is the restriction of the map

$$
\chi: \mathbb{Q}\left\langle x_{1}, x_{2}, x_{3}, \ldots\right\rangle \longrightarrow \mathbb{Q}\left[x_{1}, x_{2}, x_{3}, \ldots\right]
$$

which is the map that forgets the variables are noncommutative. Clearly this map is an algebra morphism. It follows that the restriction of this map to NCSym and Sym will also be an algebra morphism.

In addition we need to show that $(\chi \otimes \chi) \circ \Delta^{N C S y m}=\Delta^{\text {Sym }} \circ \chi$. We remark that for a given $\mu$ the number of subsets $S$ such that $\lambda\left(A_{S}\right)=\mu$ and $\lambda\left(A_{S^{c}}\right)=\nu$ with $n_{i}(\mu)+n_{i}(\nu)=n_{i}(\lambda(A))$ is equal to $\frac{\lambda(A)^{!}}{\mu^{!} \nu^{!}}$. Therefore

$$
\begin{aligned}
(\chi \otimes \chi) \circ \Delta^{N C S y m}\left(\mathbf{m}_{A}\right) & =\sum_{S \subseteq[\ell(A)]} \lambda\left(A_{S}\right)^{!} \lambda\left(A_{S^{c}}\right)^{!} m_{\lambda\left(A_{S}\right)} \otimes m_{\lambda\left(A_{S^{c}}\right)} \\
& =\sum_{\mu \uplus \nu=\lambda(A)} \mu^{!} \nu^{!}\left(\frac{\lambda(A)^{!}}{\mu^{!} \nu^{!}}\right) m_{\mu} \otimes m_{\nu} \\
& =\sum_{\mu \uplus \nu=\lambda(A)} \lambda(A)^{!} m_{\mu} \otimes m_{\nu} \\
& =\Delta^{S y m}\left(\lambda(A)^{!} m_{\lambda(A)}\right)=\Delta^{S y m}\left(\chi\left(\mathbf{m}_{A}\right)\right) .
\end{aligned}
$$

Therefore $\chi$ is also a morphism with respect to the coproduct and hence is a Hopf morphism.

There is a natural pullback of $\chi: N C S y m \rightarrow$ Sym which was considered by Rosas and Sagan in 12. They called the linear homomorphism $\tilde{\chi}:$ Sym $\rightarrow$ NCSym defined by $\tilde{\chi}\left(m_{\lambda}\right)=\frac{\lambda !}{|\lambda| !} \sum_{\lambda(A)=\lambda} \mathbf{m}_{A}$ the lifting map and showed it has the following property.

Proposition 4.4. 12, Proposition 4.1] $\chi \circ \tilde{\chi}$ is the identity map on Sym.

For our purposes, the important property of the lifting map will be from the following proposition.

\section{Proposition 4.5.}

$$
\Delta^{N C S y m} \circ \tilde{\chi}=(\tilde{\chi} \otimes \tilde{\chi}) \circ \Delta^{S y m} .
$$


Proof. Equation (6) allows us to deduce this property by direct computation.

$$
\begin{aligned}
\Delta^{N C S y m}\left(\tilde{\chi}\left(m_{\lambda}\right)\right) & =\frac{\lambda !}{|\lambda| !} \sum_{C: \lambda(C)=\lambda} \Delta^{N C S y m}\left(\mathbf{m}_{C}\right) \\
& =\frac{\lambda !}{|\lambda| !} \sum_{C: \lambda(C)=\lambda} \sum_{S \subseteq[\ell(\lambda)]} \mathbf{m}_{C_{S}} \otimes \mathbf{m}_{C_{S^{c}}} \\
& =\frac{\lambda !}{|\lambda| !} \sum_{C: \lambda(C)=\lambda} \sum_{\mu \uplus \nu=\lambda} \sum_{\substack{S \subseteq[\ell(\lambda)] \\
\lambda\left(C_{S}\right)=\mu}} \mathbf{m}_{C_{S}} \otimes \mathbf{m}_{C_{S^{c}}} \\
& =\frac{\lambda !}{|\lambda| !} \sum_{C: \lambda(C)=\lambda} \sum_{\mu \uplus \nu=\lambda} \sum_{\substack{A: \lambda(A)=\mu \\
B: \lambda(B)=\nu}} \sum_{\substack{S \subseteq[\ell(\lambda)] \\
C_{S}=A \\
C_{S c}=B}} \mathbf{m}_{A} \otimes \mathbf{m}_{B}
\end{aligned}
$$

Now to complete this computation we exchange the sums and notice that for a fixed set partitions $A$ and $B$, there are exactly $\left(\begin{array}{l}|C| \\ |A|\end{array}\right)$ different set partitions $C$ such that there is an $S \subseteq[\ell(C)]$ with $C_{S}=A$ and $C_{S^{c}}=B$

$$
\begin{aligned}
& =\frac{\lambda !}{|\lambda| !} \sum_{\mu \uplus \nu=\lambda} \sum_{\substack{A: \lambda(A)=\mu \\
B: \lambda(B)=\nu}}\left(\begin{array}{l}
|\lambda| \\
|\mu|
\end{array}\right) \mathbf{m}_{A} \otimes \mathbf{m}_{B} \\
& =\sum_{\mu \uplus \nu=\lambda} \sum_{\substack{A: \lambda(A)=\mu \\
B: \lambda(B)=\nu}} \frac{\lambda !}{|\lambda| ! \mid} \frac{|\lambda| !}{|\mu| !|\nu| !} \mathbf{m}_{A} \otimes \mathbf{m}_{B} \\
& =\sum_{\mu \uplus \nu=\lambda} \sum_{\substack{A: \lambda(A)=\mu \\
B: \lambda(B)=\nu}} \frac{\lambda !}{|\mu| !|\nu| !} \mathbf{m}_{A} \otimes \mathbf{m}_{B} .
\end{aligned}
$$

Now since $\mu \uplus \nu=\lambda$ then we have that $\lambda$ ! $=\mu$ ! $\nu$ ! and hence the equation above is equal to

$$
=\sum_{\mu \uplus \nu=\lambda} \tilde{\chi}\left(m_{\mu}\right) \otimes \tilde{\chi}\left(m_{\nu}\right)=(\tilde{\chi} \otimes \tilde{\chi}) \circ \Delta^{S y m}\left(m_{\lambda}\right)
$$

This last proposition leads us to identifying an important relationship between the algebra of NSym of noncommutative symmetric functions and the algebra of NCSym.

Theorem 4.6. Define $\mathcal{I}: N S y m \rightarrow$ NCSym by the action

$$
\mathcal{I}\left(\mathbf{h}_{n}\right)=\tilde{\chi}\left(h_{n}\right)=\sum_{A \vdash[n]} \frac{\lambda(A) !}{n !} \mathbf{m}_{A}
$$

and extend this as an algebra morphism by defining for the linear basis $\mathbf{h}_{\alpha}$,

$$
\mathcal{I}\left(\mathbf{h}_{\alpha}\right)=\tilde{\chi}\left(h_{\alpha_{1}}\right) \tilde{\chi}\left(h_{\alpha_{2}}\right) \cdots \tilde{\chi}\left(h_{\alpha_{\ell(\alpha)}}\right) .
$$

$\mathcal{I}$ is a Hopf morphism and $\mathcal{I}$ an inclusion map so that NSym is a natural subalgebra of NCSym. 
Before proceeding with the proof of the theorem we introduce an important lemma. For each $\alpha$ a composition of $n$, we have a canonical corresponding set partition,

$$
A(\alpha)=\left\{1,2, \ldots, \alpha_{1}, \alpha_{1}+1, \ldots, \alpha_{1}+\alpha_{2}, \cdots, \alpha_{1}+\cdots+\alpha_{\ell(\alpha)-1}, \ldots,|\alpha|\right\} .
$$

Lemma 4.7. The coefficient of $\mathbf{m}_{A(\beta)}$ in $\mathcal{I}\left(\mathbf{h}_{\alpha}\right)$ is equal to $(\alpha \cup \beta) ! / \alpha$ ! where $\alpha \cup \beta$ is the composition with descent set equal to $D(\alpha) \cup D(\beta)$ and $\alpha !=\alpha_{1} ! \alpha_{2} ! \cdots \alpha_{\ell(\alpha)}$ ! for a composition $\alpha$.

Proof. Note that $\mathcal{I}\left(\mathbf{h}_{\alpha}\right)=\mathcal{I}\left(\mathbf{h}_{\tilde{\alpha}}\right) \mathcal{I}\left(\mathbf{h}_{\alpha_{\ell(\alpha)}}\right)$ where $\tilde{\alpha}=\left(\alpha_{1}, \alpha_{2}, \ldots, \alpha_{\ell(\alpha)-1}\right)$. Let $\tilde{B}=$ $\left.A(\beta)\right|_{\{1, \ldots,|\tilde{\alpha}|\}}$ which corresponds to a composition $\tilde{\beta}$ and $\bar{B}=\operatorname{st}\left(\left.A(\beta)\right|_{\{|\tilde{\alpha}|+1, \ldots,|\alpha|\})}\right)$. When $\mathbf{m}_{A(\beta)}$ arises as the coefficient of $\mathcal{I}\left(\mathbf{h}_{\alpha}\right)$ the coefficient will be the coefficient of $\mathbf{m}_{\tilde{B}}$ in $\mathcal{I}\left(\mathbf{h}_{\tilde{\alpha}}\right)$ times the coefficient of $\mathbf{m}_{\bar{B}}$ in $\mathcal{I}\left(\mathbf{h}_{\alpha_{\ell(\alpha)}}\right)$. By induction on the number of parts of $\alpha$ we can assume that this coefficient is $\frac{(\tilde{\alpha} \cup \tilde{\beta}) !}{\tilde{\alpha} !} \frac{\lambda(\bar{B}) !}{\alpha_{\ell(\alpha)} !}=\frac{(\tilde{\alpha} \cup \tilde{\beta}) ! \lambda(\bar{B}) !}{\alpha !}=\frac{(\alpha \cup \beta) !}{\alpha !}$.

Proof of Theorem. Proposition 4.5 says that

$$
\begin{aligned}
\Delta^{\operatorname{NCSym}}\left(\mathcal{I}\left(\mathbf{h}_{n}\right)\right) & =(\tilde{\chi} \otimes \tilde{\chi}) \circ \Delta^{\text {Sym }}\left(h_{n}\right) \\
& =\sum_{k=0}^{n} \tilde{\chi}\left(h_{k}\right) \otimes \tilde{\chi}\left(h_{n-k}\right) \\
& =(\mathcal{I} \otimes \mathcal{I}) \circ \Delta^{N S y m}\left(\mathbf{h}_{n}\right) .
\end{aligned}
$$

Clearly we have that $\mathcal{I}\left(\mathbf{h}_{\alpha} \mathbf{h}_{\beta}\right)=\mathcal{I}\left(\mathbf{h}_{\alpha}\right) \mathcal{I}\left(\mathbf{h}_{\beta}\right)$ so we know that $\mathcal{I}$ is a Hopf morphism. In order to show that $\mathcal{I}$ is an inclusion of NSym into NCSym we need to show that the generators of $N S y m, \mathcal{I}\left(\mathbf{h}_{n}\right)$, are algebraically independent. This is equivalent to showing that the elements $\mathcal{I}\left(\mathbf{h}_{\alpha_{1}}\right) \mathcal{I}\left(\mathbf{h}_{\alpha_{2}}\right) \cdots \mathcal{I}\left(\mathbf{h}_{\alpha_{\ell(\alpha)}}\right)$ are linearly independent.

In order to show that $\mathcal{I}\left(\mathbf{h}_{\alpha}\right)$ are linearly independent, it suffices to examine the minor of coefficients of $\mathbf{m}_{A(\beta)}$ in $\mathcal{I}\left(\mathbf{h}_{\alpha}\right)$ and show that the determinant of this minor is nonzero. The coefficient of $\mathbf{m}_{A(\beta)}$ in $\mathcal{I}\left(\mathbf{h}_{\alpha}\right)$ is $(\alpha \cup \beta)$ !/ $\alpha$ ! by Lemma 4.7 .

The proof follows by showing that the $2^{n-1} \times 2^{n-1}$ determinant of the matrix $[(\alpha \cup \beta) !]_{\alpha, \beta \models n}$ is nonzero. In Theorem 4.8 below we compute that this matrix has a nonzero determinant (in fact we compute it explicitly) and hence conclude that $\mathcal{I}$ is an inclusion.

By writing the first few matrices and their determinants gives a clue on how to show that it has a nonzero determinant. Begin by ordering the compositions in lexicographic order so that $(11)<(2),(111)<(12)<(21)<(3)$, and $(1111)<(112)<(121)<(13)<(211)<$ $(22)<(31)<(4)$ are the order of the indices of the matrices below.

$$
\begin{gathered}
\left|\begin{array}{ll}
1 & 1 \\
1 & 2
\end{array}\right|=1 \\
\left|\begin{array}{llll}
1 & 1 & 1 & 1 \\
1 & 2 & 1 & 2 \\
1 & 1 & 2 & 2 \\
1 & 2 & 2 & 6
\end{array}\right|=3
\end{gathered}
$$




$$
\left|\begin{array}{llllllll}
1 & 1 & 1 & 1 & 1 & 1 & 1 & 1 \\
1 & 2 & 1 & 2 & 1 & 2 & 1 & 2 \\
1 & 1 & 2 & 2 & 1 & 1 & 2 & 2 \\
1 & 2 & 2 & 6 & 1 & 2 & 2 & 6 \\
1 & 1 & 1 & 1 & 2 & 2 & 2 & 2 \\
1 & 2 & 1 & 2 & 2 & 4 & 2 & 4 \\
1 & 1 & 2 & 2 & 2 & 2 & 6 & 6 \\
1 & 2 & 2 & 6 & 2 & 4 & 6 & 24
\end{array}\right|=117=3^{2} \cdot 13
$$

The next two values of this determinant are $2915757=3^{5} \cdot 13^{2} \cdot 71$ and $458552896435013913=$ $3^{12} \cdot 13^{5} \cdot 71^{2} \cdot 461$. Although the sequence of determinants is not familiar, the factors which appear in it are. The sequence $1,1,3,13,71,461, \ldots$ are found in the OLEIS [14 as sequence A003319, the permutations of $n$ with no global descents. A global descent is a value $k$ such that $\pi_{i}>\pi_{j}$ for all $i \leq k$ and $j>k$. The number of these can be calculated with the recurrence $a_{1}=1$ and for $n>1$,

$$
a_{n}=n !-\sum_{i=1}^{n-1} a_{i}(n-i) ! .
$$

The permutations with no global descents arise in the Hopf algebra of permutations due to Malvenuto-Reutenauer as the primitive elements/generators of the Hopf algebra [1]. This begs an explanation of why these numbers should arise in this computation. The expression for the determinant is summarized in the following theorem.

\section{Theorem 4.8.}

$$
\operatorname{det}|(\alpha \cup \beta) !|_{\alpha, \beta \models n}=\prod_{\alpha \models n} \prod_{i=1}^{\ell(\alpha)} a_{\alpha_{i}}
$$

where $a_{n}$ is the number of permutations of $n$ with no global descents.

One could prove this theorem by induction using the identity for a block matrix (see [10])

$$
\operatorname{det}\left[\begin{array}{cc}
A & B \\
C & D
\end{array}\right]=\operatorname{det}(A) \operatorname{det}\left(D-C A^{-1} B\right)
$$

and the recursive structure of the matrix $[(\alpha \cup \beta) !]_{\alpha, \beta \models n}$. Instead we present a proof suggested to us by A. Lascoux [8] which makes the formula of Theorem 4.8 transparent.

Proof. It is easy to see that any permutation can be decomposed uniquely as a concatenation of permutations with no global descents. For example, 465312 can be decomposed as 465 . $3 \cdot 12$.

Using this decomposition, we associate to any permutation a composition. For instance to $465 \cdot 3 \cdot 12$ we associate the composition $(3,1,2)$. Therefore, the set of all permutations in $\mathfrak{S}_{n}$ can be partitioned into a disjoint union of subclasses indexed by compositions.

In addition, the cardinality of the class indexed by $\alpha \models n$ is $a_{\alpha}:=a_{\alpha_{1}} a_{\alpha_{2}} \cdots a_{\alpha_{\ell(\alpha)}}$, where $a_{j}$ is the number of permutations with no global descents. 
We conclude that

$$
\sum_{\alpha \models n} a_{\alpha}=n !
$$

An analogous statement holds for any Young subgroup of $\mathfrak{S}_{n}$. To wit,

$$
\sum_{\beta \leq \alpha} a_{\beta}=\alpha !
$$

with $\leq$ representing the standard refinement order on compositions. Now our notation for $\alpha \cup \beta$ means that for $\eta \leq(\alpha \cup \beta)$ that $\eta \leq \alpha$ and $\eta \leq \beta$ and so using the notation $([\operatorname{true}])=1$ and $([$ false $]=0$ we have the expression

$$
\left.(\alpha \cup \beta) !=\sum_{\eta \leq(\alpha \cup \beta)} a_{\eta}=\sum_{\eta}([\eta \leq \alpha])(\eta \eta \beta]\right) a_{\eta} .
$$

Let $\mathbb{D}$ represent the $2^{n-1} \times 2^{n-1}$ diagonal matrix indexed by compositions $\eta$ with $a_{\eta}$ the entries along the diagonal. Also let $\mathbb{C}=\left[([\beta \leq \alpha)]_{\alpha, \beta \models n}\right.$, a matrix with entry 1 at $(\alpha, \beta)$ if $\beta \leq \alpha$ and 0 otherwise. Now look at the $(\alpha, \beta)$ entry in the product $\mathbb{C D} \mathbb{C}^{T}$. This will be

$$
\sum_{\delta, \theta \models n}([\delta \leq \alpha])([\delta=\theta]) a_{\theta}([\theta \leq \beta])=\sum_{\delta \models n}\left([\delta \leq \alpha]([\delta \leq \beta]) a_{\delta}=(\alpha \cup \beta) !\right.
$$

We conclude that $\mathbb{C D} \mathbb{C}^{T}=[(\alpha \cup \beta) !]_{\alpha, \beta \models n}$ and hence $\operatorname{det}[(\alpha \cup \beta) !]_{\alpha, \beta \models n}=\operatorname{det} \mathbb{D}$ (since $\operatorname{det} \mathbb{C}=1)$. This demonstrates (17) since $\mathbb{D}$ is a diagonal matrix with determinant equal to $\prod_{\alpha \models n} a_{\alpha}$.

Remark 1. NSym is also generated by the analogs of the power and elementary bases of the symmetric functions and there are formulas for expressing these into the $\mathbf{h}$-basis. The map $\mathcal{I}$ is not unique since we could just as easily lift these other bases (as we defined $\left.\mathcal{I}\left(\mathbf{h}_{n}\right)=\tilde{\chi}\left(h_{n}\right)\right)$ and by direct computation one can verify that these other inclusions of NSym in NCSym are not the same as $\mathcal{I}$. For example, $\mathbf{e}_{3}=\mathbf{h}_{3}-\mathbf{h}_{12}-\mathbf{h}_{21}+\mathbf{h}_{111}$ and

$$
\mathcal{I}\left(\mathbf{e}_{3}\right)=\frac{1}{6} \mathbf{m}_{\{1,2,3\}}+\frac{1}{3} \mathbf{m}_{\{13,2\}}-\frac{1}{6} \mathbf{m}_{\{12,3\}}-\frac{1}{6} \mathbf{m}_{\{1,23\}} \neq \tilde{\chi}\left(e_{3}\right)=\frac{1}{6} \mathbf{m}_{\{1,2,3\}} .
$$

Therefore the inclusion that we present here is not unique, but once we fix a set of generators of Sym there is a natural embedding of NSym to NCSym.

We conclude this section with a summary of these results stating that the Hopf algebra morphisms which relate NSym, NCSym and Sym can be drawn in a commutative diagram.

Theorem 4.9. The following diagram commutes and all maps are Hopf morphisms.

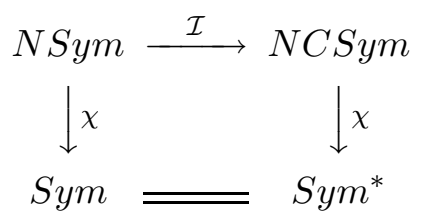




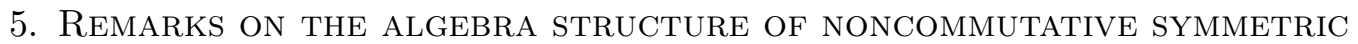 FUNCTIONS}

There are other relationships between NCSym and NSym that are worth considering but are not as structured because they only hold on the level of algebras and do not respect the coproduct. First, we shall examine a graded algebra isomorphism between the graded algebras NSym and $\mathrm{NCSym}_{2}$.

This algebra isomorphism implies that the structure constants for $\mathrm{NCSym}_{2}$ with respect to the monomial basis coincide with the structure constants for NSym in the ribbon Schur basis, which are know to be related to the representation theory of the Hecke algebra at $q=0$, see for example [7.

In general, the structure constants of $\mathrm{NCSym}_{n}$ with respect to the monomial basis (as well as those of NCSym) are also nonnegative integers. A natural question to ask is whether the representation theoretical interpretations of $\mathrm{NCSym}_{2}$ can be extended to NCSym, as well as to its specializations $\mathrm{NCSym}_{n}$, for each value of $n$.

The number of set partitions of $[n]$ with at most two blocks is $2^{n-1}$. Therefore, there is a bijection between set partitions of $[n]$ with at most two parts and compositions of $n$. Compositions are the indexing set of the algebra NSym of subsection 4.2 and so it is natural to look for a connection through this structure. In fact, this observation gives us a way of relating $\mathrm{NCSym}_{2}$ and NSym. That is, NSym is isomorphic, as an algebra, to $\mathrm{NCSym}_{2}$.

To any set partition $A=\left\{A_{1}, A_{2}\right\}$, we associate the ribbon shape obtained by reading numbers $1,2, \cdots, n$ sequentially, and placing the $(i+1)^{s t}$ box to the right of the $i^{\text {th }}$ box if $i$ and $i+1$ are in the same block of $A$, or placing the $(i+1)^{s t}$ box immediately below the $i^{\text {th }}$ box otherwise. For instance, the ribbon associated to $A=\{1245,3678\}$ is

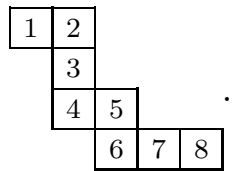

Note that we are placing numbers inside the boxes for the sake of clarity only. We denote by $c(A)$ the composition of $n$ obtained by recording the lengths of the horizontal segments in the corresponding ribbon. In our example, $c(A)=(2,1,2,3)$.

Following [17, we define a second and very important basis for NSym, the ribbon Schur functions. Recall that the set of all compositions of $n$ is equipped with the reverse refinement order of the descent sets, denoted by $\leq$. For instance, $(2,2,1) \leq(4,1)$. The ribbon Schur functions $\left(\mathbf{R}_{\alpha}\right)$ are defined by the following expression:

$$
\mathbf{R}_{\alpha}=\sum_{\beta \leq \alpha}(-1)^{\ell(\alpha)-\ell(\beta)} \mathbf{h}_{\alpha}
$$

Let $\iota: N C S y m_{2} \mapsto N S y m$ by the linear homomorphism such that

$$
\iota\left(\mathbf{m}_{\{A, B\}}\right)=\mathbf{R}_{c(\{A, B\})},
$$

where $c(\{A, B\})$ denotes the composition corresponding to $\{A, B\}$ under the bijection just stated. 
Proposition 5.1. The map $\iota: N_{C S y m} \mapsto N S y m$ is an isomorphism of algebras.

Proof. The monomials in $\mathrm{NCSym}_{2}$ multiply according to the following rule

$$
\mathbf{m}_{\left\{A_{1}, A_{2}\right\}} \mathbf{m}_{\left\{B_{1}, B_{2}\right\}}=\mathbf{m}_{\left\{A_{1} \cup\left(B_{1}+|A|\right), A_{2} \cup\left(B_{2}+|A|\right)\right\}}+\mathbf{m}_{\left\{A_{1} \cup\left(B_{2}+|A|\right), A_{2} \cup\left(B_{1}+|A|\right)\right\}} \cdot
$$

For instance, if $A=\{1346,2578\}$ and $B=\{12,345\}$, then $B+8=\{910,111213\}$, and the two terms in the product of $\mathbf{m}_{A}\left[x_{1}, x_{2}\right]$ and $\mathbf{m}_{B}\left[x_{1}, x_{2}\right]$ are indexed by

$$
\left\{A_{1} \cup\left(B_{1}+8\right), A_{2} \cup\left(B_{2}+8\right)\right\}=\{1346910,2578111213\}
$$

and

$$
\left\{A_{1} \cup\left(B_{2}+8\right), A_{2} \cup\left(B_{1}+8\right)\right\}=\{1346111213,2578910\} .
$$

On the other hand, it is well known that the ribbon Schur functions multiply as $\mathbf{R}_{\alpha} \mathbf{R}_{\beta}=$ $\mathbf{R}_{\alpha \triangleright \beta}+\mathbf{R}_{\alpha \cdot \beta}$, where $\alpha \triangleright \beta$ is the composition obtained by adding the last part of $\alpha$ to the first part of $\beta$, and $\alpha \cdot \beta$ is the composition obtained by concatenation. Hence, multiplying ribbons $\mathbf{R}_{\alpha}$ and $\mathbf{R}_{\beta}$ is equivalent to placing the first box of $\mathbf{R}_{\beta}$ next to the last box of $\mathbf{R}_{\alpha}$ either vertically or horizontally.

To finish our argument, note that if $\alpha=c\left(\left\{A_{1}, A_{2}\right\}\right)$, and $\beta=c\left(\left\{B_{1}, B_{2}\right\}\right)$. Then, $\alpha \triangleright \beta=$ $c\left(\left\{A_{1} \cup\left(B_{1}+|A|\right), A_{2} \cup\left(B_{2}+|A|\right)\right\}\right)$ and $\alpha \cdot \beta=c\left(\left\{A_{1} \cup\left(B_{2}+|A|\right), A_{2} \cup\left(B_{1}+|A|\right)\right\}\right)$. This is best done looking at our running example and noticing that joining the last row of $\alpha$ and the first row of $\beta$ corresponds to joining blocks $A_{1}$ and $B_{1}$ together, and placing the the first row of $\beta$ below the last row of $\alpha$ corresponds to joining blocks $A_{1}$ and $B_{2}$ together (or vice-versa, depending on the position of largest element in $\{A, B\}$ ).

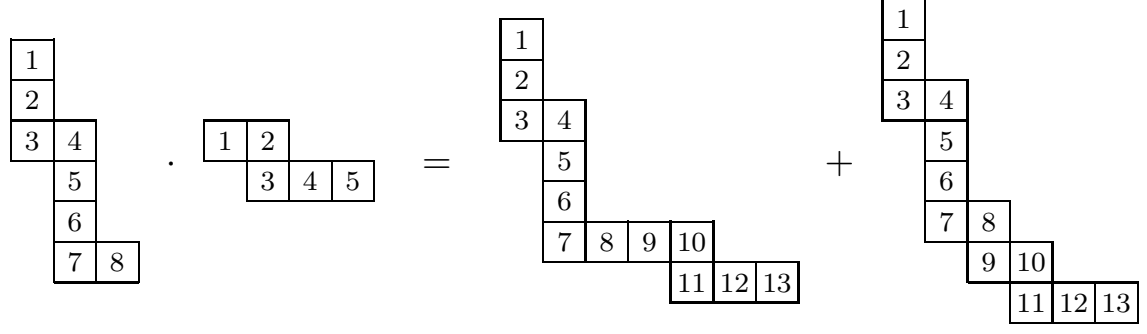

This algebra isomorphism also arises as a quotient space. Define the two sided ideal of $N C S y m$ generated by the monomials $\left\{\mathbf{m}_{A} \mid \ell(A) \geq 3\right\}$ as $I_{3}$. Notice that if $A$ has $\ell(A) \geq 3$ then every term in the product $\mathbf{m}_{A} \mathbf{m}_{B}$ will be indexed by a set partition of length greater than or equal to 3 and hence the ideal is linearly spanned by this set of monomials as well. We have then that $N C S y m / I_{3} \simeq N S y m$ since the quotient will be linearly spanned by the $\mathbf{m}_{A}$ for $\ell(A) \leq 2$.

There is another closely related copy of NSym sitting inside NCSym. Let $\alpha$ be a composition, and let $A(\alpha)$ be the corresponding canonical set partition from equation (15). We define $M_{\alpha}$ to be the sum of all monomials in NCSym indexed by those set partitions $A$ that can be obtained from $A(\alpha)$ by gluing nonconsecutive blocks. For instance, if $\alpha=(2,1,3,2)$, then $A(\alpha)=\{12,3,456,78\}$, and we can only obtain the following five set partitions $\{12,3,456,78\},\{12456,3,78\},\{12,378,456\},\{12456,378\},\{1278,3,456\}$. A second way of describing $M_{\alpha}$ is as the sum of all monomials in $\mathbb{Q}\left\langle x_{1}, x_{2}, \ldots\right\rangle$ whose exponents 
are given by composition $\alpha$. For instance, $M_{(2,1,3,2)}=\sum_{i \neq j \neq k \neq l} x_{i}^{2} x_{j} x_{k}^{3} x_{l}^{2}$ where in the sum we allow any of the possibilities of $i=k, i=\ell$ or $j=\ell$.

Proposition 5.2. The map $\zeta: N$ Sym $\rightarrow$ NCSym by $\zeta\left(R_{\alpha}\right)=M_{\alpha}$ is an injective algebra homomorphism.

Proof. The map $\zeta$ is clearly injective, hence it suffices to show that $\zeta\left(\mathbf{R}_{\alpha} \mathbf{R}_{\beta}\right)=\zeta\left(\mathbf{R}_{\alpha}\right) \zeta\left(\mathbf{R}_{\beta}\right)$. We have that

$$
\begin{aligned}
\zeta\left(\mathbf{R}_{\alpha} \mathbf{R}_{\beta}\right) & =\zeta\left(\mathbf{R}_{\alpha \triangleright \beta}+\mathbf{R}_{\alpha \cdot \beta}\right) \\
& =\sum_{i_{1} \neq \cdots \neq i_{\ell(\alpha)}=j_{1} \neq \cdots \neq j_{\ell(\beta)}} x_{i_{1}}^{\alpha_{1}} \cdots x_{i_{\ell}(\alpha)}^{\left.\alpha_{\ell(\alpha)}\right)} x_{j_{1}}^{\beta_{1}} \cdots x_{j_{\ell}(\beta)}^{\beta_{\ell(\beta)}} \\
& +\sum_{i_{1} \neq \cdots \neq i_{\ell(\alpha)} \neq j_{1} \neq \cdots \neq j_{\ell(\beta)}}^{\alpha_{i_{1}}^{\alpha_{1}} \cdots x_{i_{\ell}(\alpha)}^{\alpha_{\ell(\alpha)}} x_{j_{1}}^{\beta_{1}} \cdots x_{j_{\ell}(\beta)}^{\left.\beta_{\ell(\beta)}\right)}} \\
& =\left(\sum_{i_{1} \neq \cdots \neq i_{\ell(\alpha)}} x_{i_{1}}^{\alpha_{1}} \cdots x_{i_{\ell}(\alpha)}^{\alpha_{\ell(\alpha)}}\right)\left(\sum_{j_{1} \neq \cdots \neq j_{\ell(\beta)}} x_{j_{1}}^{\beta_{1}} \cdots x_{j_{\ell}(\beta)}^{\beta_{\ell(\beta)}}\right) \\
& =\zeta\left(\mathbf{R}_{\alpha}\right) \zeta\left(\mathbf{R}_{\beta}\right) .
\end{aligned}
$$

The following observation, due to Florent Hivert [6], shows us that NSym is also a quotient of NCSym.

When $A$ is not equal to $A(\alpha)$ for any $\alpha$ then we will say that $A$ has crossings. We remark that if $A$ has crossings then so will every term in the expansion of $\mathbf{m}_{A} \mathbf{m}_{B}$. Consider the two sided ideal $I$ generated by all $\mathbf{m}_{A}$ such that $A$ has crossings. This ideal is then linearly spanned by all $\mathbf{m}_{A}$ such that $A$ has crossings.

Now consider the quotient $N C S y m / I$. It is linearly spanned by the basis $\mathbf{m}_{A(\alpha)}$ for $\alpha$ a composition. The proof that the elements $\mathcal{I}\left(\mathbf{h}_{\alpha}\right)$ are all linearly independent also shows that they will be linearly independent in the quotient $N C S y m / I$.

\section{Corollary 5.3}

$$
N S y m \simeq N C S y m / I .
$$

as algebras. The isomorphism is given explicitly as $\rho: N C S y m \rightarrow N$ Sym by

$$
\rho\left(\mathbf{m}_{A}\right)=\left\{\begin{array}{cl}
\mathbf{m}_{A} & \text { if } A \text { has no crossings } \\
0 & \text { otherwise }
\end{array} .\right.
$$

A computation of $\Delta^{N C S y m} \circ \rho \circ \mathcal{I}\left(\mathbf{h}_{3}\right)$ and $(\rho \otimes \rho) \circ \Delta^{N C S y m} \circ \mathcal{I}\left(\mathbf{h}_{3}\right)$ shows that these spaces are not isomorphic as Hopf algebras since $I$ is not a Hopf ideal.

\section{The Harmonics with Respect to the Hausdorff Derivative.}

We give an elegant characterization of the space of harmonics in noncommuting variables with respect to the Hausdorff derivative in terms of the free Lie algebra. We will require 
some basic definitions and results for which we refer the reader to [11] for references and their proofs.

A Lie algebra over $\mathbb{Q}$ is a $\mathbb{Q}$-module $\mathcal{L}$, together with a bilinear mapping

$$
\begin{aligned}
\mathcal{L} \times \mathcal{L} & \rightarrow \mathcal{L} \\
(x, y) & \mapsto[x, y]
\end{aligned}
$$

called the Lie bracket. This bracket must satisfy two identities, $[x, y]=-[y, x]$ and $[x,[y, z]]+[y,[z, x]]+[z,[x, y]]=0$. Subalgebras of Lie algebras, homomorphisms and modules are defined as usual for Lie algebras. Any associative algebra $\mathcal{A}$ over $\mathbb{Q}$ acquires a natural structure of a Lie algebra when $[x, y]$ is defined by $[x, y]=x y-y x$. The free Lie algebra can be realized as the linear span of the minimal set of polynomials in $\mathbb{Q}\left\langle X_{n}\right\rangle$ which include the variables $\left\{x_{1}, x_{2}, \ldots, x_{n}\right\}$ and is closed under the bracket operation.

For a Lie algebra $\mathcal{L} \subseteq \mathbb{Q}\left\langle X_{n}\right\rangle$ with the natural bracket operation, the enveloping algebra of $\mathcal{L}$ is the subalgebra of $\mathbb{Q}\left\langle X_{n}\right\rangle$ generated by the elements of $\mathcal{L}$ under the concatenation product.

Let $\mathcal{L}=\mathcal{L}\left(X_{n}\right)$ be the free Lie algebra generated by the noncommutative alphabet $X_{n}=$ $\left\{x_{1}, x_{2}, \cdots, x_{n}\right\}$, and let $\mathcal{L}^{\prime}=[\mathcal{L}, \mathcal{L}]$ be the Lie subalgebra generated by the brackets $[P, Q]$ where both $P$ and $Q$ are in $\mathcal{L}$. Let $\mathcal{A}^{\prime}$ be the enveloping algebra of $\mathcal{L}^{\prime}$. In particular, $\mathcal{L}=\mathcal{L}^{\prime}+\mathbb{Q} X_{n}$, where $\mathbb{Q} X_{n}$ denotes the linear polynomials.

We want to characterize the harmonics of the symmetric group in noncommuting variables. Recall that in the commutative setting the harmonics are defined as the set of solutions for the system of PDE obtained by looking at symmetric functions as differential operators.

Our goal is to compute the harmonics of the symmetric group in the noncommutative setting. To this end, we should start by defining what we mean by the derivative of a noncommutative polynomial. We first focus our attention on the Hausdorff derivative, the most common definition for derivative in the noncommutative setting.

Let $w$ be a monomial in $\mathbb{Q}\langle X\rangle$, that is, a word. The Hausdorff derivative of $w$ with regard to the letter $x$ is defined as the sum of all subwords $w^{\prime}$ obtained from $w$ by deleting an occurrence of letter $x$, and then extended by linearity. For instance, $\partial_{x} x y x^{2} y=y x^{2} y+$ $2 x y x y$, and $\partial_{x}[x, y]=\partial_{x}(x y)-\partial_{x}(y x)=0$.

The following theorem can be found in 11 and characterizes the elements of $\mathcal{A}^{\prime}$ as the elements of $\mathbb{Q}\left\langle X_{n}\right\rangle$ that are killed by each derivation.

Proposition 6.1. (11])

$$
\bigcap_{x \in X_{n}} \operatorname{ker} \partial_{x}=\mathcal{A}^{\prime}
$$

For any polynomial $f \in \mathbb{Q}\left\langle X_{n}\right\rangle$, we will denote by $f\left(\partial_{X_{n}}\right)$ the linear differential operator formed by replacing each of the monomials $x_{i_{1}} x_{i_{2}} \cdots x_{i_{k}}$ by the differential $\partial_{x_{i_{1}}} \partial_{x_{i_{2}}} \cdots \partial_{x_{i_{k}}}$. Note that $\partial_{x} \partial_{y}=\partial_{y} \partial_{x}$ and so we have that the operator $\mathbf{m}_{A}\left(\partial_{X_{n}}\right)$ acts up to constant as $m_{\lambda(A)}\left(\partial_{X_{n}}\right)$. More precisely, $\mathbf{m}_{A}\left(\partial_{X_{n}}\right)\left(f\left(X_{n}\right)\right)=\chi\left(\mathbf{m}_{A}\right)(\partial)\left(f\left(X_{n}\right)\right)$.

Definition 1. Let $X_{n}=\left\{x_{1}, x_{2}, \ldots, x_{n}\right\}$ be a finite noncommuting alphabet, the harmonics with respect to the Hausdorff derivative are defined as the space of solutions of the system 
of PDEs

$$
f\left(\partial_{X_{n}}\right) Q\left(X_{n}\right)=0
$$

for all $f \in \mathrm{NCSym}_{n}$ without constant term. We denote the solution space by $\mathrm{MHar}_{n}$.

Theorem 6.2. (Poincaré-Birkhoff-Witt) Let $\mathcal{L}$ be a Lie algebra and consider $\mathcal{L}$ as a vector space with a totally ordered basis $\left(w_{i}\right)_{i \in I}$. Let $\mathcal{A}_{0}$ be its enveloping algebra and $\varphi_{0}: \mathcal{L} \rightarrow \mathcal{A}_{0}$ be the natural Lie algebra homomorphism. Then $\mathcal{A}_{0}$ is a vector space over $\mathbb{Q}$ with basis $\varphi_{0}\left(w_{i_{1}}\right) \ldots \varphi_{0}\left(w_{i_{n}}\right)$, where $n \geq 0, i_{1}, \ldots, i_{n} \in I$, and $i_{1} \geq \ldots \geq i_{n}$.

Let $\mathcal{L}$ be the free Lie algebra in the variables $X_{n}$. Take a basis $\mathcal{B}^{\prime}$ of $\mathcal{L}^{\prime}=[\mathcal{L}, \mathcal{L}]$, then the linear polynomials $\mathbb{Q} X_{n}$ satisfy $\mathcal{L}=\mathcal{L}^{\prime} \oplus \mathbb{Q} X_{n}$ and

$$
\mathcal{B}=\mathcal{B}^{\prime} \cup X_{n}
$$

is a basis for $\mathcal{L}$. Next, order the basis in such a way that the elements of $\mathcal{B}^{\prime}$ are strictly bigger than the elements of $X_{n}$.

The enveloping algebra of the free Lie algebra $\mathcal{L}$ is $\mathbb{Q}\left\langle X_{n}\right\rangle$. Therefore, the theorem of Poincaré-Birkhoff-Witt implies that decreasing products of elements of $\mathcal{B}$ form a basis of $\mathbb{Q}\left\langle X_{n}\right\rangle$. Moreover, since $\mathcal{A}^{\prime}$ is the enveloping algebra of $\mathcal{L}^{\prime}$, the theorem of Poincaré-BirkhoffWitt also implies that decreasing products of $\mathcal{B}^{\prime}$ are a basis of $\mathcal{A}^{\prime}$. We also know that decreasing products of $X_{n}$ are isomorphic to $\mathbb{Q}\left[X_{n}\right]$. We conclude that, as vector spaces,

$$
\mathbb{Q}\left\langle X_{n}\right\rangle \simeq \mathcal{A}^{\prime} \otimes \mathbb{Q}\left[X_{n}\right] \simeq \mathcal{A}^{\prime}\left[X_{n}\right]
$$

Furthermore, this isomorphism is compatible with derivations $\partial_{a}$. That is, for a $P\left(X_{n}\right) \in$ $\mathbb{Q}\left\langle X_{n}\right\rangle$ where $P\left(X_{n}\right)=\sum_{i} b_{i} f_{i}\left(X_{n}\right)$ and $b_{i} \in \mathcal{A}^{\prime}$ and $f_{i}\left(X_{n}\right) \in \mathbb{Q}\left[X_{n}\right]$, we have

$$
\partial_{a} P\left(X_{n}\right)=\sum_{i} b_{i} \partial_{a} f_{i}\left(X_{n}\right)
$$

for $a \in X_{n}$. This follows because $\partial_{a}\left(\mathcal{A}^{\prime}\right)=0$.

We have from this discussion the following theorem.

Theorem 6.3. Let $\mathcal{H}_{n}$ be the classical harmonics. That is,

$$
\mathcal{H}_{n}=\left\{f\left(X_{n}\right) \in \mathbb{Q}\left[X_{n}\right]: p\left(\partial_{X_{n}}\right) f\left(X_{n}\right)=0 \text { for all } p\left(X_{n}\right) \in \text { Sym }_{n} \text { with } p(0)=0\right\} .
$$

Then, as vector spaces,

$$
\operatorname{MHar}_{n} \simeq \mathcal{A}^{\prime} \otimes \mathcal{H}_{n}
$$

Moreover, Chevalley [4] showed that $\mathcal{H}_{n}$ the linear span of derivatives of the Vandermonde polynomial

$$
\Delta_{n}=\prod_{1 \leq i<j \leq n}\left(x_{i}-x_{j}\right)=\sum_{\pi \in \mathfrak{S}_{n}} \operatorname{sgn}(\pi) x_{n}^{\pi_{1}-1} x_{n-1}^{\pi_{2}-1} \cdots x_{1}^{\pi_{n}-1} .
$$

Note that from the existence of the isomorphism (20) and the classical characterization for the harmonics in the commutative case, we obtain that $N \Delta_{n} \in M \operatorname{Har}_{n}$, where $N \Delta_{n}$ is the noncommutative Vandermonde, defined as

$$
N \Delta_{n}=\sum_{\pi \in \mathfrak{S}_{n}} \operatorname{sgn}(\pi) x_{n}^{\pi_{1}-1} x_{n-1}^{\pi_{2}-1} \cdots x_{1}^{\pi_{n}-1}
$$


It is interesting to note that all other possible noncommutative Vandermondes (obtained by fixing an order in the variables) are also harmonics, but one suffices to describe this space. To see this, we only need to order $X_{n}$ in all possible ways before applying the PoincaréBirkhoff-Witt theorem. Likewise, we have that each derivative of $N \Delta_{n}$ is in MHar ${ }_{n}$. Therefore, $\operatorname{Span}_{\partial}\left[N \Delta_{n}\right] \subseteq \operatorname{MHar}_{n}$. Theorem 6.3 implies that MHar $_{n}$ is equal to the $\mathcal{A}^{\prime}$-module generated by $N \Delta_{n}$ and all its derivatives.

A famous theorem due to Chevalley says that the ring of polynomials is isomorphic to the tensor product of its invariants times its coinvariants (that in the commutative case are show to be isomorphic to the harmonics).

Theorem 6.4 (Chevalley [4]). As $\mathfrak{S}_{n}$-modules,

$$
\mathcal{H}_{n} \otimes \operatorname{Sym}_{n} \simeq \mathbb{Q}\left[X_{n}\right] .
$$

We conclude a mixed commutative/noncommutative version of Chevalley's theorem which holds on the level of vector spaces which we derive from the results above. But to get the isomorphism as $\mathfrak{S}_{n}$-module we need some more tools.

We first define on $\mathbb{Q}\left\langle X_{n}\right\rangle$ a commutative product. The shuffle product, denoted by $\sqcup$, is the bilinear operation recursively defined as follow. Given variables $x, y$ and monomials $v, w \in \mathbb{Q}\left\langle X_{n}\right\rangle$,

$$
1 \sqcup u=u \sqcup \sqcup 1=u \quad \text { and } \quad x u \sqcup y v=x(u \sqcup y v)+y(x u \sqcup v) .
$$

This is a well known commutative product on $\mathbb{Q}\left\langle X_{n}\right\rangle$. It is clear that the forgetful map $\chi: \mathbb{Q}\left\langle X_{n}\right\rangle \rightarrow \mathbb{Q}\left[X_{n}\right]$ acts as

$$
\chi\left(x_{i_{1}} \sqcup x_{i_{2}} \sqcup \cdots \sqcup x_{i_{k}}\right)=k ! x_{i_{1}} x_{i_{2}} \cdots x_{i_{k}} .
$$

Define now $\tilde{p}_{k}=\sum_{i=1}^{n} x_{i} \sqcup x_{i} \sqcup \cdots \sqcup x_{i}=\mathbf{m}_{\{[k]\}}\left[X_{n}\right]$ where the variable $x_{i}$ is shuffled with itself $k$ times. For $\lambda=\left(\lambda_{1}, \lambda_{2}, \ldots, \lambda_{\ell(\lambda)}\right) \vdash m$ a partition of the integer $m$ we let $\tilde{p}_{\lambda}=\tilde{p}_{\lambda_{1}} \sqcup \cdots \sqcup \tilde{p}_{\lambda_{\ell(\lambda)}}$. We then have that

$$
\chi\left(\tilde{p}_{\lambda}\right)=\lambda ! p_{\lambda_{1}} p_{\lambda_{2}} \cdots p_{\lambda_{\ell(\lambda)}},
$$

where $p_{k}=\sum_{i=1}^{n} x_{i}^{k} \in S y m_{n}$ is the classical power sum symmetric polynomial. If we denote by $\widetilde{\operatorname{Sym}}_{n} \subseteq \mathbb{Q}\left\langle X_{n}\right\rangle$ the vector space spanned by the $\tilde{p}_{\lambda}$ with $1 \leq \lambda_{i} \leq n$.

Lemma 6.5. As graded $\mathfrak{S}_{n}$-modules, $\widetilde{\operatorname{Sym}}_{n} \simeq \operatorname{Sym}_{n}$.

Proof. It is well known that $S y m_{n}=\mathbb{Q}\left[p_{1}, p_{2}, \ldots, p_{n}\right]$. The map $\chi$ in equation (21) restricted to $\widetilde{\operatorname{Sym}}_{n}$ gives us a surjective linear map $\chi: \widetilde{\operatorname{Sym}}_{n} \rightarrow S y m_{n}$. This map preserves the degree of the polynomial, so we can restrict our attention to the homogeneous component of degree $m, \widetilde{\operatorname{Sym}}_{n}^{m}$. Since the product $\sqcup$ is commutative, $\operatorname{dim}\left(\widetilde{\operatorname{Sym}}_{n}^{m}\right) \leq \operatorname{dim}\left(\operatorname{Sym} m_{n}^{m}\right)$ the number of partitions $\lambda \vdash m$ with $1 \leq \lambda_{i} \leq n$. Hence $\chi: \widetilde{S y m}_{n} \rightarrow S y m_{n}$ is an isomorphism of graded vector spaces. Since each element of $\widetilde{\operatorname{Sym}}_{n}$ is $\mathfrak{S}_{n}$ invariant (as is $S y m_{n}$ ), $\widetilde{\operatorname{Sym}}_{n}$ and Sym $_{n}$ are isomorphic as $\mathfrak{S}_{n}$ modules as well.

Let us denote by $N C S y m_{n}^{+}$the set of $f \in N C S y m_{n}$ without constant term. Recall that the map $\chi: \mathrm{NCSym}_{n} \rightarrow \mathrm{Sym}_{n}$ is surjective and also that the Hausdorff derivative commutes. 
We thus have $f(\partial)=\chi(f)(\partial)$ for all $f \in \mathbb{Q}\left\langle X_{n}\right\rangle$. Combining these remarks, we get

$$
\begin{aligned}
\operatorname{MHar}_{n} & =\left\{P \in \mathbb{Q}\left\langle X_{n}\right\rangle \mid f(\partial) P=0, \forall f \in N C \text { Sym }_{n}^{+}\right\} \\
& =\left\{P \in \mathbb{Q}\left\langle X_{n}\right\rangle \mid \chi(f)(\partial) P=0, \forall f \in N C S y m_{n}^{+}\right\} \\
& =\left\{P \in \mathbb{Q}\left\langle X_{n}\right\rangle \mid p_{k}(\partial) P=0,1 \leq k \leq n\right\} .
\end{aligned}
$$

Now let $\langle$,$\rangle denote the scalar product on \mathbb{Q}\left\langle X_{n}\right\rangle$ for which the monomials forms an orthonormal basis. For all variable $x \in X_{n}$ and monomials $u, v \in \mathbb{Q}\left\langle X_{n}\right\rangle$ we easily see that

$$
\langle x \sqcup u, v\rangle=\left\langle u, \partial_{x} v\right\rangle .
$$

Finally let $\left\langle\tilde{p}_{k}: 1 \leq k \leq n\right\rangle_{\amalg} \subseteq \mathbb{Q}\left\langle X_{n}\right\rangle$ denote the ideal generated using the shuffle product. That is

$$
\left\langle\tilde{p}_{k}: 1 \leq k \leq n\right\rangle_{\sqcup}=\left\{\sum_{k=1}^{n} \tilde{p}_{k} \sqcup q_{k} \mid q_{k} \in \mathbb{Q}\left\langle X_{n}\right\rangle\right\} .
$$

\section{Lemma 6.6.}

$$
\operatorname{MHar}_{n}=\left\langle\tilde{p}_{k}: 1 \leq k \leq n\right\rangle \stackrel{\perp}{\perp}
$$

Proof. If $P \in$ MHar $_{n}$ then for all $1 \leq k \leq n$ we have $p_{k}(\partial) P=0$. Given any $F \in\left\langle\tilde{p}_{k}\right.$ : $1 \leq k \leq n\rangle_{\sqcup,}, F=\sum_{k=1}^{n} p_{k} \sqcup q_{k}$ where $q_{k} \in$ polys and we calculate

$$
\begin{aligned}
\langle F, P\rangle & =\sum_{k=1}^{n} \sum_{i=1}^{n}\left\langle x_{i} \sqcup \cdots \sqcup x_{i} \sqcup q_{k}, P\right\rangle \\
& =\sum_{k=1}^{n} \sum_{i=1}^{n}\left\langle q_{k}, \partial_{x_{i}}^{k} P\right\rangle=\sum_{k=1}^{n}\left\langle q_{k}, p_{k}(\partial) P\right\rangle=0,
\end{aligned}
$$

where we have use the identity (22) $k$ times in the summands. Hence $P \in M H a r_{n}$ implies $P \in\left\langle\tilde{p}_{k}: 1 \leq k \leq n\right\rangle \stackrel{\perp}{ }$. Conversely if $P \notin M \operatorname{Mar}_{n}$, then there is a $1 \leq k \leq n$ such that $p_{k}(\partial) P \neq 0$. This means we can find $q \in \mathbb{Q}\left\langle X_{n}\right\rangle$ such that

$$
0 \neq\left\langle q, p_{k}(\partial) P\right\rangle=\sum_{i=1}^{n}\left\langle q, \partial_{x_{i}}^{k} P\right\rangle=\left\langle\tilde{p}_{k} \sqcup q, P\right\rangle
$$

and conclude that $P \notin\left\langle\tilde{p}_{k}: 1 \leq k \leq n\right\rangle \stackrel{\perp}{\perp}$.

At this point, we have shown that $\mathbb{Q}\left\langle X_{n}\right\rangle=\operatorname{MHar}_{n} \oplus\left\langle\tilde{p}_{k}: 1 \leq k \leq n\right\rangle_{\amalg}$. This gives us for any $G \in \mathbb{Q}\left\langle X_{n}\right\rangle$

$$
G=P+\sum_{k=1}^{n} \tilde{p}_{k} \sqcup q_{k}=1 \sqcup P+\sum_{k=1}^{n} \tilde{p}_{k} \sqcup q_{k},
$$

where $P \in \mathrm{MHar}_{n}$ and $\operatorname{deg}\left(q_{k}\right)<\operatorname{deg}(G)$. If we repeat the use of equation (23) recursively on the $q_{k}$ we get that

$$
G=\sum_{\lambda} \tilde{p}_{\lambda} \sqcup P_{\lambda}
$$


where the sum runs over $\lambda=\left(\lambda_{1}, \ldots, \lambda_{\ell(\lambda)}\right)$ such that $1 \leq \lambda_{i} \leq n$ and $P_{\lambda} \in$ MHar $_{n}$. Also by convention we allow $\lambda=()$ and $\tilde{p}_{()}=1$. This equation shows that the graded linear map

$$
\psi: \widetilde{\operatorname{Sym}}_{n} \otimes \operatorname{MHar}_{n} \rightarrow \mathbb{Q}\left\langle X_{n}\right\rangle,
$$

defined by $\psi\left(\tilde{p}_{\lambda} \otimes P\right)=\tilde{p}_{\lambda} \sqcup P$, is surjective.

Theorem 6.7. As graded $\mathfrak{S}_{n}$-modules,

$$
\operatorname{Sym}_{n} \otimes \operatorname{MHar}_{n} \simeq \mathbb{Q}\left\langle X_{n}\right\rangle .
$$

Proof. By equation (20) and Theorems 6.3 and 6.4 we have

$$
\text { Sym }_{n} \otimes \text { MHar }_{n} \simeq \text { Sym }_{n} \otimes \mathcal{H}_{n} \otimes \mathcal{A}^{\prime} \simeq \mathbb{Q}\left[X_{n}\right] \otimes \mathcal{A}^{\prime} \simeq \mathbb{Q}\left\langle X_{n}\right\rangle .
$$

as vector spaces. Combined with Lemma 6.5] and the surjectivity of $\psi$, this shows that

$$
\psi \circ\left(\chi^{-1} \otimes i d\right): \operatorname{Sym}_{n} \otimes \operatorname{MHar}_{n} \rightarrow \widetilde{\operatorname{Sym}}_{n} \otimes \operatorname{MHar}_{n} \rightarrow \mathbb{Q}\left\langle X_{n}\right\rangle
$$

is surjective, and hence an isomorphism of vector spaces.

To view the result as an isomorphism of $\mathfrak{S}_{n}$-modules, we first need to make sure that $\mathrm{MHar}_{n}$ is indeed an $\mathfrak{S}_{n}$-module. This follows from the fact that for any $P \in M H a r_{n}$, any $\sigma \in \mathfrak{S}_{n}$ and for all $1 \leq k \leq n, p_{k}(\partial) \sigma(P)=\sigma\left(p_{k}(\partial) P\right)=0$ and thus $\sigma(P) \in$ MHar $_{n}$. We

already know that $\chi$ restricted to $\widetilde{S y m}_{n}$ is a morphism of $\mathfrak{S}_{n}$-modules. It thus remains to show that $\psi \circ\left(\chi^{-1} \otimes i d\right)$ is also a morphism of $\mathfrak{S}_{n}$-modules. For this let $\sigma \in \mathfrak{S}_{n}$ :

$$
\sigma \circ \psi\left(\tilde{p}_{\lambda} \otimes P\right)=\sigma\left(\tilde{p}_{\lambda} \sqcup P\right)=\left(\sigma \tilde{p}_{\lambda}\right) \sqcup(\sigma P)=\psi\left(\left(\sigma \tilde{p}_{\lambda}\right) \otimes(\sigma P)\right)=\psi \circ(\sigma \otimes \sigma)\left(\tilde{p}_{\lambda} \otimes P\right) .
$$

Since both $\tilde{p}_{\lambda}$ and $p_{\lambda}$ are both $\mathfrak{S}_{n}$ invariant, $\sigma \circ \psi \circ\left(\chi^{-1} \otimes i d\right)=\psi \circ\left(\chi^{-1} \otimes i d\right) \circ(\sigma \otimes \sigma)$ and our proof is then complete.

\section{Noncommutative InVARIANTS OF THE SYMmETRIC GROUP}

In classical invariant theory of the symmetric group (see [9, 16]) the ring of symmetric polynomials in $n$ (commuting) variables is free. In particular it is a polynomial ring with $n$ generators, one in each degree. In [18], Wolf was the first to study $N C S y m_{n}$ as invariants in noncommuting variables. Her main theorem shows that the space of noncommutative invariants of the symmetric group is also free.

In her proof, it is not obvious how to construct the generators and the combinatorics of set partitions is not fully developed. In particular, it is not clear what the Hilbert series of the invariant polynomial ring in $n$ noncommutative variables is. In the final section of this article we will need her result and the associated Hilbert series. We thus present it here along with a constructive proof.

Given two set partitions $A=\left\{A_{1}, \ldots, A_{k}\right\} \vdash[n]$ and $B=\left\{B_{1}, \ldots, B_{\ell}\right\} \vdash[m]$, we define

$$
A \circ B=\left\{\begin{array}{ll}
\left\{A_{1} \cup\left(B_{1}+n\right), \ldots, A_{k} \cup\left(B_{k}+n\right),\left(B_{k+1}+n\right), \ldots,\left(B_{\ell}+n\right)\right\} & \text { if } k \leq \ell \\
\left\{A_{1} \cup\left(B_{1}+n\right), \ldots, A_{\ell} \cup\left(B_{\ell}+n\right), A_{\ell+1}, \ldots, A_{k}\right\} & \text { if } k>\ell
\end{array} .\right.
$$


Recall that the parts of $A$ and $B$ are ordered according to the minimum elements in each part. For example, if $A=\{13,2\}$ and $B=\{1,2,3\}$ then $A \circ B=\{134,25,6\}$ and $B \circ A=$ $\{146,25,3\}$. We note that $\ell(A \circ B)=\max (\ell(A), \ell(B))$.

If $A=B \circ C$ for $B$ and $C$ nonempty set partitions, then we say that $A$ splits. If it is not possible to split $A$, then we say that it is nonsplitable. By convention, only non-empty set partitions are nonsplitable.

Example 7.1. For $n=3$. the list of all set partitions is

$$
\begin{array}{lll}
\{123\}=\{1\} \circ\{1\} \circ\{1\} & \{1,23\} \text { nonsplitable } & \{13,2\}=\{1,2\} \circ\{1\} \\
\{12,3\}=\{1\} \circ\{1,2\} & \{1,2,3\} \text { nonsplitable } &
\end{array}
$$

As we remarked in Section 3 a basis for $\operatorname{NCSym}_{n}$ is given by $\left\{\mathbf{m}_{A}\left[X_{n}\right]\right\}_{\ell(A) \leq n}$. Consider the set $X_{n}$ as an alphabet where $x_{1}<x_{2}<\cdots<x_{n}$. A monomial in these noncommutative variables can be viewed as a word in the alphabet $X_{n}$. Given $A$ such that $\ell(A) \leq n$, we order the monomials of $\mathbf{m}_{A}\left[X_{n}\right]$ by lexicographic order and denote by $\operatorname{LT}\left(\mathbf{m}_{A}\left[X_{n}\right]\right)$ the smallest monomial in $\mathbf{m}_{A}\left[X_{n}\right]$. For example, $L T\left(\mathbf{m}_{\{14,25,3\}}\left[X_{6}\right]\right)=x_{1} x_{2} x_{3} x_{1} x_{2}$. In general, the $k$ th variable of $L T\left(\mathbf{m}_{A}\left[X_{n}\right]\right)$ is $x_{i}$ exactly when $k \in A_{i}$ and the parts of $A$ are ordered according to the minimum elements in each part.

Lemma 7.2. For any set partitions $A$ and $B$ with at most $n$ parts, we have

$$
\operatorname{LT}\left(\mathbf{m}_{A}\left[X_{n}\right] \mathbf{m}_{B}\left[X_{n}\right]\right)=\operatorname{LT}\left(\mathbf{m}_{A}\left[X_{n}\right]\right) \operatorname{LT}\left(\mathbf{m}_{B}\left[X_{n}\right]\right) .
$$

Proof. This is a direct consequence of the following well known fact about lexicographic order. Given four words (monomials) $u_{1}, u_{2}, v_{1}, v_{2}$ in the alphabet $X_{n}$ such that $u_{1} \leq_{l e x} v_{1}$ and $u_{2} \leq_{l e x} v_{2}$, then $u_{1} u_{2} \leq_{l e x} v_{1} v_{2}$. So the smallest term of $\mathbf{m}_{A}\left[X_{n}\right] \mathbf{m}_{B}\left[X_{n}\right]$ is the product of the smallest term of $\mathbf{m}_{A}\left[X_{n}\right]$ with the smallest term of $\mathbf{m}_{B}\left[X_{n}\right]$, and all terms have coefficient equal to 1 .

We now proceed to show the main results of Wolf.

Proposition 7.3. $\mathrm{NCSym}_{n}$ is freely generated as an algebra by

$$
\left\{\mathbf{m}_{A}\left[X_{n}\right]: \ell(A) \leq n \text { and } A \text { is nonsplitable }\right\} .
$$

Proof. Let $B \vdash[m]$ be a set partition such that $\ell=\ell(B) \leq n$. Let $1 \leq k \leq m$ be the smallest integer such that

$$
\min \left(B_{i} \cap[k]^{c}\right) \leq \min \left(B_{j} \cap[k]^{c}\right) \text { for each } 1 \leq i<j \leq \ell(B)
$$

where we use the convention that $[k]^{c}=\{k+1, \ldots, m\}$ and $\min (\emptyset)=\infty$.

Let $B^{(1)}=\left\{B_{i} \cap[k]: i \leq r\right\} \vdash[k]$, then by the choice of $k, B^{(1)}$ is nonsplitable.

If $k=m$, then $B=B^{(1)}$ is nonsplitable, otherwise $k<m$ and $\tilde{B}=\left\{\left(B_{i} \cap\{k+1, \ldots, m\}\right)-k\right.$ : $i \leq \ell\} \vdash[m-k]$ with $B=B^{(1)} \circ \tilde{B}$. Repeating this process recursively, we obtain a unique decomposition of $B$ into nonsplitable set partitions:

$$
B=B^{(1)} \circ B^{(2)} \circ \cdots \circ B^{(s)} .
$$


Now consider the following expansion:

$$
W_{B}\left[X_{n}\right]:=\mathbf{m}_{B^{(1)}}\left[X_{n}\right] \mathbf{m}_{B^{(2)}}\left[X_{n}\right] \cdots \mathbf{m}_{B^{(s)}}\left[X_{n}\right]=\sum_{D \vdash[m]} c_{D} \mathbf{m}_{D}\left[X_{n}\right] .
$$

Since $L T\left(\mathbf{m}_{B^{(1)}}\left[X_{n}\right] \cdots \mathbf{m}_{B^{(s)}}\left[X_{n}\right]\right)=L T\left(\mathbf{m}_{B}\left[X_{n}\right]\right)$ we have that $c_{B}=1$ and $c_{D}=0$ whenever $L T\left(\mathbf{m}_{D}\left[X_{n}\right]\right)<_{\text {lex }} L T\left(\mathbf{m}_{B}\left[X_{n}\right]\right)$. This implies that the change of basis matrix between the basis $\left\{\mathbf{m}_{B}\left[X_{n}\right]: \ell(B) \leq n\right\}$ and the set $\mathcal{W}=\left\{W_{B}\left[X_{n}\right]: \ell(B) \leq n\right\}$ is upper triangular and therefore $\mathcal{W}$ is a linear basis of $N_{C S y m}$. We now remark that $\mathcal{W}$ is also a basis of the free noncommutative algebra generated by the set $\left\{\mathbf{m}_{A}\left[X_{n}\right]: \ell(A) \leq\right.$ $n$ and $A$ is nonsplitable\} and this concludes the proof.

Let $S_{m, k}$ denote the number of set partitions of $m$ with exactly $k$ parts (the Stirling numbers of the second kind). Then the number of set partition of $m$ with at most $n$ parts is $\sum_{k=1}^{n} S_{m, k}$. We thus have that

$$
B_{n}(q)=\sum_{m \geq 0} \operatorname{dim}_{m}\left(\operatorname{NCSym}_{n}\right) q^{m}=\sum_{m \geq 0} \sum_{i=1}^{n} S_{m, i} q^{m},
$$

where $\operatorname{dim}_{m}\left(\mathrm{NCSym}_{n}\right)$ is the dimension of the homogeneous component of degree $m$ in $\mathrm{NCSym}_{n}$. Let $w_{m, n}$ be the number of nonsplitable set partitions of $m$ with at most $n$ parts and let $W_{n}(q)=\sum_{m \geq 0} w_{m, n} q^{m}$. A direct consequence of the previous theorem is that $B_{n}(q)=\left(1-W_{n}(q)\right)^{-1}$. Thus

$$
W_{n}(q)=1-\frac{1}{B_{n}(q)}
$$

As we will require the use of these numbers later, we include a table of the values of $w_{m, n}$ for $1 \leq m, n \leq 8$.

\begin{tabular}{c|cccccccc}
$m / n$ & 1 & 2 & 3 & 4 & 5 & 6 & 7 & 8 \\
\hline 1 & 1 & 1 & 1 & 1 & 1 & 1 & 1 & 1 \\
2 & 0 & 1 & 1 & 1 & 1 & 1 & 1 & 1 \\
3 & 0 & 1 & 2 & 2 & 2 & 2 & 2 & 2 \\
4 & 0 & 1 & 5 & 6 & 6 & 6 & 6 & 6 \\
5 & 0 & 1 & 13 & 21 & 22 & 22 & 22 & 22 \\
6 & 0 & 1 & 34 & 78 & 91 & 92 & 92 & 92 \\
7 & 0 & 1 & 89 & 297 & 406 & 425 & 426 & 426 \\
8 & 0 & 1 & 233 & 1143 & 1896 & 2119 & 2145 & 2146
\end{tabular}

Notice that the differences between adjacent entries in this tables is the number of generators of a fixed length as described by Wolf.

\section{The CoInvariants of the Symmetric Group}

Let $X_{n}$ be an alphabet with $n$ letters, and let $\mathbb{Q}\left\langle X_{n}\right\rangle$ be the corresponding ring of noncommutative polynomials. 
We denote by $\left\langle N C S y m_{n}^{+}\right\rangle=\mathcal{L}\left\{P\left(X_{n}\right) \mathbf{m}_{A}\left[X_{n}\right] \mid k \geq 1, A \vdash[k], P\left(X_{n}\right) \in \mathbb{Q}\left\langle X_{n}\right\rangle\right\}$ the left ideal of $\mathbb{Q}\left\langle X_{n}\right\rangle$ generated by all elements of $N C S y m$ without constant term. The coinvariant algebra of the symmetric group in noncommutative variables will be defined as the quotient:

$$
\mathbb{Q}\left\langle X_{n}\right\rangle /\left\langle N C \text { Sym }_{n}^{+}\right\rangle \text {. }
$$

To find a linear basis of the space $\mathbb{Q}\left\langle X_{n}\right\rangle /\left\langle N C S y m_{n}^{+}\right\rangle$we use some standard techniques of the theory of languages, that can be found in [3]. We start by introducing some definitions.

Let $L^{*}$ be the free monoid generated by $L$, an alphabet for the monoid. A suffix set is a subset $C$ of $L^{*}$ such that for all $u$ and $v$ in $L^{*}$, if $v, u v$ are both in $C$ implies that $u=\emptyset$.

A subset $P$ of $L^{*}$ is prefix closed (resp. suffix closed) if $u v \in P$ (resp. $v u \in P$ ) implies $u \in P$ for all words $u$ and $v$. There is a bijection between suffix sets and suffix closed sets. To a suffix set $C$ is associated the suffix-closed set $P=L^{*} \backslash L^{*} C$, that is the set of words which do not end with an element of $C$. Moreover, $L^{*}=P C^{*}$.

For a polynomial $P\left(X_{n}\right) \in \mathbb{Q}\left\langle X_{n}\right\rangle$, the leading term with respect to the lexicographic order will be denoted $\operatorname{LT}\left(P\left(X_{n}\right)\right.$ ) (without the leading coefficient, hence $\operatorname{LT}\left(P\left(X_{n}\right)\right.$ ) will be an element of $X_{n}^{*}$ ).

Noncommutative monomial symmetric functions indexed by set partitions have the property that $\left\{L T\left(\mathbf{m}_{A}\left[X_{n}\right]\right) \mid A\right.$ a set partition $\}$ is a prefix closed set. That is, any prefix $u$ of $L T\left(\mathbf{m}_{A}\left[X_{n}\right]\right)$ is the leading term of $\mathbf{m}_{B}\left[X_{n}\right]$ for some set partition $B$. In particular $B$ will be equal to $A$ restricted to $\{1,2, \ldots,|u|\}$. For example, if $A=\{13,246,5\}$ has leading term $x_{1} x_{2} x_{1} x_{2} x_{3} x_{2}$ and the heads of these monomials are $x_{1}, x_{1} x_{2}, x_{1} x_{2} x_{1}, x_{1} x_{2} x_{1} x_{2}, x_{1} x_{2} x_{1} x_{2} x_{3}$ corresponding to the leading terms of $\mathbf{m}_{\{1\}}\left[X_{n}\right], \mathbf{m}_{\{1,2\}}\left[X_{n}\right], \mathbf{m}_{\{13,2\}}\left[X_{n}\right], \mathbf{m}_{\{13,24\}}\left[X_{n}\right]$, and $\mathbf{m}_{\{13,24,5\}}\left[X_{n}\right]$.

We established in the previous section that the $W_{A}\left[X_{n}\right]$ of equation (26) for $A$ a set partition with $\ell(A) \leq n$ are a basis for $N_{C S y m}$ we will use this to show that the polynomials $u \mathbf{m}_{A}\left[X_{n}\right]$ for $A$ nonsplitable and $u \in X_{n}^{*}$ form a basis for $\left\langle N C S y m_{n}^{+}\right\rangle$.

Proposition 8.1. Let

$$
C=\left\{L T\left(\mathbf{m}_{A}\left[X_{n}\right]\right) \mid A \text { nonsplitable, } \ell(A) \leq n\right\} .
$$

$C$ is a suffix set of the language $X_{n}^{*}$.

Proof. Suppose $u$ and $v u$ are both in $C$ and $v \neq \emptyset$. Then since $v u=L T\left(\mathbf{m}_{A}\left[X_{n}\right]\right)$ for $A$ nonsplitable then $v=L T\left(\mathbf{m}_{B}\left[X_{n}\right]\right)$ for some nonempty set partition $B$ since it is the head of the monomial $L T\left(\mathbf{m}_{A}\left[X_{n}\right]\right)$. Because $u=L T\left(\mathbf{m}_{C}\left[X_{n}\right]\right)$ for some nonsplitable set partition $C$, we can conclude that $A=B \circ C$, but this contradicts that $A$ is nonsplitable.

For any left ideal $I$ of $\mathbb{Q}\left\langle X_{n}\right\rangle$ we note that the set $M_{I}=\{L T(f) \mid f \in I\}$ is a left monomial ideal. That is, for each $v \in M_{I}$ and for each $u \in X_{n}^{*}, u v \in M_{I}$.

Now we are ready to describe precisely the quotient $\mathbb{Q}\left\langle X_{n}\right\rangle /\left\langle N C S y m_{n}^{+}\right\rangle$. Consider the set of leading terms of $\left\langle N C S y m_{n}^{+}\right\rangle, M_{\left\langle N C S y m_{n}^{+}\right\rangle}=\left\{L T(f) \mid f \in\left\langle N C S y m_{n}^{+}\right\rangle\right\}$, which is a left 
monomial ideal. We note that

$$
\begin{aligned}
M_{\left\langle N C S y m_{n}^{+}\right\rangle} & =\left\{v L T\left(\mathbf{m}_{A}\left[X_{n}\right]\right) \mid v \in X_{n}^{*}, A \text { set partition }\right\} \\
& =\left\{v L T\left(\mathbf{m}_{A}\left[X_{n}\right]\right) \mid v \in X_{n}^{*}, A \text { nonsplitable set partition }\right\} .
\end{aligned}
$$

That is, $M_{\left\langle N C S y m_{n}^{+}\right\rangle}=X_{n}^{*} C$ where $C$ is given Proposition 8.1 . We conclude then by the correspondence between suffix closed sets and suffix sets that

$$
M_{\left\langle N C S y m_{n}^{+}\right\rangle}^{c}=X_{n}^{*} \backslash M_{\left\langle N C S y m_{n}^{+}\right\rangle}=X_{n}^{*} \backslash X_{n}^{*} C
$$

is suffix closed.

Proposition 8.2. The set

$$
\left\{u \mathbf{m}_{A}\left[X_{n}\right] \mid u \in X_{n}^{*}, A \text { nonsplitable set partition }\right\}
$$

is a linear basis of $\left\langle N C S y m_{n}^{+}\right\rangle$and any element of $w \in M_{\left\langle N C S y m_{n}^{+}\right\rangle}=X_{n}^{*} C$ can be decomposed uniquely as $w=u v$ where $u \in X_{n}^{*}$ and $v \in C$.

Proof. Assume $u v=u^{\prime} v^{\prime} \in X_{n}^{*} C$ with $v, v^{\prime} \in C$. Without loss of generality assume $v=$ $w v^{\prime} \in C$. Since $C$ is a suffix set then $w=\emptyset$ and hence $v=v^{\prime}$ and $u=u^{\prime}$. Therefore the decomposition of $u v \in M_{\left\langle N_{C S y m}^{+}\right\rangle}$is unique.

Next consider the set $\left\{u \mathbf{m}_{A}\left[X_{n}\right] \mid u \in X_{n}^{*}, A\right.$ nonsplitable set partition $\}$. Since the leading terms of the elements of this set are all distinct, they are linearly independent. This set must also span the ideal $\left\langle N C S y m_{n}^{+}\right\rangle$because every element of the form $v W_{A}\left[X_{n}\right] \in\left\langle N C S y m_{n}^{+}\right\rangle$ is in the linear span of this set and the $v W_{A}\left[X_{n}\right]$ are certainly a spanning set of the ideal.

Proposition 8.3. $M_{\left\langle N C S y m_{n}^{+}\right\rangle}^{c}$ is a basis for $\mathbb{Q}\left\langle X_{n}\right\rangle /\left\langle N C S y m_{n}^{+}\right\rangle$.

Proof. First we show that $M_{\left\langle N C S y m_{n}^{+}\right\rangle}^{c}$ spans the vector space. Since the words of $X_{n}^{*}$ span $\mathbb{Q}\left\langle X_{n}\right\rangle$, it suffices to show that for $v \in X_{n}^{*}$,

$$
v \equiv \sum_{u \in M_{\left\langle N C S y m_{n}^{+}\right\rangle}^{c}} a_{u} u \bmod \left\langle N C S y m_{n}^{+}\right\rangle .
$$

Assume that $v$ is the smallest such monomial which is not a linear combination of $u \in$ $M_{\left\langle N C S y m_{n}^{+}\right\rangle}^{c}$. Since $v \notin M_{\left\langle N C S y m_{n}^{+}\right\rangle}^{c}$, then $v \in X_{n}^{*} C=M_{\left\langle N C S y m_{n}^{+}\right\rangle}$and so $v=u L T\left(\mathbf{m}_{A}\left[X_{n}\right]\right)$ for some nonsplitable set partition $A$. Now $v-u \mathbf{m}_{A}\left[X_{n}\right]$ is equal to a sum of terms which are smaller than $v$ in lexicographic order and hence are equivalent to a linear combination of elements of $M_{\langle N C S y m}^{c}{ }_{n}^{*}$.

The monomials in $M_{\left\langle N C S y m_{n}^{+}\right\rangle}^{c}$ are also linearly independent since if we assume that

$$
P\left(X_{n}\right)=\sum_{u \in M_{\left\langle N C S y m_{n}^{+}\right\rangle}^{c}} a_{u} u \equiv 0 \bmod \left\langle N C S y m_{n}^{+}\right\rangle,
$$

then $P\left(X_{n}\right) \in\left\langle N C\right.$ Sym $\left.m_{n}^{+}\right\rangle$and hence $L T\left(P\left(X_{n}\right)\right) \in M_{\left\langle N C S y m_{n}^{+}\right\rangle}$. Since the leading term of $P\left(X_{n}\right)$ is one of the monomials of $M_{\left\langle N C S y m_{n}^{+}\right\rangle}^{c}$, the only way this can happen is if $P\left(X_{n}\right)=$ 0 .

We conclude from Propositions 8.2 and 8.3 the following corollary. 
Corollary 8.4. The dimension of the subspace of degree $k$ in $\mathbb{Q}\left\langle X_{n}\right\rangle /\left\langle N C S y m_{n}^{+}\right\rangle$is equal to

$$
n^{k}-\sum_{i \leq k} w_{i, n} n^{k-i}
$$

where $w_{m, k}$ is the number of nonsplitable set partitions of size $m$ and with length less than or equal to $k$.

Define the following three generating functions:

$$
\begin{aligned}
T_{n}(q) & :=\sum_{k} \operatorname{dim}_{k}\left(\mathbb{Q}\left\langle X_{n}\right\rangle\right) q^{k}=\frac{1}{1-n q} \\
B_{n}(q) & :=\sum_{k} \operatorname{dim}_{k}\left(N C \operatorname{Sym}_{n}\right) q^{k}=\frac{1}{1-W_{n}(q)}=\frac{1}{1-\sum_{k \geq 0} w_{k, n} q^{n}} \\
C_{n}(q) & :=\sum_{k} \operatorname{dim}_{k}\left(\mathbb{Q}\left\langle X_{n}\right\rangle /\left\langle N C \operatorname{Sym}_{n}^{+}\right\rangle\right) q^{k}=\sum_{k \geq 0}\left(n^{k}-\sum_{i \leq k} w_{i, n} n^{k-i}\right) q^{k}
\end{aligned}
$$

Observe that we have the relationship $T_{n}(q)=B_{n}(q) C_{n}(q)$ by the following calculation.

$$
\begin{aligned}
B_{n}(q) C_{n}(q) & =B_{n}(q)\left(\sum_{n \geq 0} n^{k} q^{k}\right)-B_{n}(q)\left(\sum_{k \geq 0} n^{k} q^{k}\right)\left(\sum_{k \geq 0} w_{k, n} q^{k}\right) \\
& =B_{n}(q)\left(\sum_{n \geq 0} n^{k} q^{k}\right)\left(1-\sum_{k \geq 0} w_{k, n} q^{k}\right) \\
& =\sum_{n \geq 0} n^{k} q^{k}=\frac{1}{1-n q}=T_{n}(q) .
\end{aligned}
$$

We conclude that as graded vector spaces

$$
\mathbb{Q}\left\langle X_{n}\right\rangle /\left\langle N C \text { Sym }_{n}^{+}\right\rangle \otimes N C \text { Sym }_{n} \simeq \mathbb{Q}\left\langle X_{n}\right\rangle .
$$

As mentioned in the introduction, the twisted derivative provides a second definition of derivation in $\mathbb{Q}\left\langle X_{n}\right\rangle$ (see for example [1] ). It is defined as

$$
d_{a}(w)=\left\{\begin{array}{cl}
w^{\prime} & \text { if } w=a w^{\prime} \\
0 & \text { otherwise }
\end{array} .\right.
$$

We can show that space of noncommutative coinvariants of the symmetric group is isomorphic the space of harmonics of the symmetric group with respect to the twisted derivative.

Recall that the scalar product is defined with the monomials as an orthonormal basis. The twisted derivative has the property analogous to equation (22) for the Hausdorff derivative. For $x \in X_{n}$ and $u, v \in \mathbb{Q}\left\langle X_{n}\right\rangle$,

$$
\langle x u, v\rangle=\left\langle u, d_{x} v\right\rangle
$$

In particular, for $P, Q \in \mathbb{Q}\left\langle X_{n}\right\rangle$,

$$
\langle P, Q\rangle=\left.P\left(d_{X_{n}}\right) \tau(Q)\right|_{x_{1}=x_{2}=\cdots=x_{n}=0}
$$


where $\tau$ be the operator on $\mathbb{Q}\left\langle X_{n}\right\rangle$ that any monomial is sent to the monomial obtained by reading its entries from right-to-left.

Definition 2. Let $X_{n}=\left\{x_{1}, x_{2}, \cdots, x_{n}\right\}$ be a finite noncommuting alphabet. The harmonics of the symmetric group, with respect to the twisted derivative, are defined as the space of solutions for the system of PDEs:

$$
f\left(d_{X_{n}}\right) Q\left(X_{n}\right)=0
$$

for all $f \in N_{C S y m}$ without constant terms. We denote them as $N C H a r_{n}$.

\section{Lemma 8.5.}

$$
\operatorname{NCHar}_{n}=\left\langle\operatorname{NCSym}_{n}^{+}\right\rangle^{\perp}
$$

Proof. By definition, it is immediate that $\mathrm{NCHar}_{n} \subseteq\left\langle N C S y m_{n}^{+}\right\rangle^{\perp}$.

Suppose that $f \in\left\langle N C \operatorname{Sym}_{n}^{+}\right\rangle^{\perp}$. Then, for all $P$ in $\left\langle N C S y m_{n}^{+}\right\rangle$,

$$
\left.P\left(d_{X_{n}}\right) f\left(X_{n}\right)\right|_{x_{1}=x_{2}=\cdots=x_{n}=0}=0 .
$$

We claim that this implies that $P\left(d_{X_{n}}\right) f\left(X_{n}\right)=0$. Suppose this is not the case. Then, let $u$ be the smallest monomial in lexicographic order that appears with nonzero coefficient in $P\left(d_{X_{n}}\right) f\left(X_{n}\right)$. Since $P$ is in the left ideal $\left\langle N C S y m_{n}^{+}\right\rangle$, so is $u P$. But by construction $(u P)\left(d_{X_{n}}\right) f\left(X_{n}\right) \neq 0$. Contradiction. Hence, NCHar $_{n}=\left\langle N C \text { Sym }_{n}^{+}\right\rangle^{\perp}$.

Now we proceed as we did before in the case of $M H a r_{n}$. We have shown that $\mathbb{Q}\left\langle X_{n}\right\rangle=$ $\mathrm{NCHar}_{n} \oplus\left\langle\mathrm{NCSym}_{n}^{+}\right\rangle$and by Proposition 8.2 any $G\left(X_{n}\right) \in \mathbb{Q}\left\langle X_{n}\right\rangle$ can be expressed uniquely as

$$
G\left(X_{n}\right)=f\left(X_{n}\right)+\sum_{A} P_{A}\left(X_{n}\right) \mathbf{m}_{A}\left[X_{n}\right]
$$

where the sum is over nonsplitable set partitions $A, f\left(X_{n}\right) \in N C H a r_{n}$, and $P_{A}\left(X_{n}\right) \in$ $\mathbb{Q}\left\langle X_{n}\right\rangle$ is of degree strictly smaller than the degree of $G$. This procedure can be repeated recursively on $P_{A}\left(X_{n}\right)$ and the products of $\mathbf{m}_{A}\left[X_{n}\right]$ expanded in terms of other basis elements for NCSym so that

$$
G\left(X_{n}\right)=\sum_{A} f_{A}\left(X_{n}\right) \mathbf{m}_{A}\left[X_{n}\right]
$$

where the sum is over all set partitions $A$ of size smaller than or equal to the degree of $G\left(X_{n}\right)$ and each $f_{A}\left(X_{n}\right) \in N C H a r_{n}$. This reduction is unique and so the map $\bar{\psi}$ : $N C \operatorname{Nar}_{n} \otimes \mathrm{NCSym}_{n} \rightarrow \mathbb{Q}\left\langle X_{n}\right\rangle$ defined as the linear extension of the map $\bar{\psi}\left(f\left(X_{n}\right) \otimes\right.$ $\left.P\left[X_{n}\right]\right)=f\left(X_{n}\right) P\left[X_{n}\right]$ is an isomorphism of vector spaces.

Proposition 8.6. As graded $\mathfrak{S}_{n}$-modules,

$$
N \operatorname{NHar}_{n} \simeq \mathbb{Q}\left\langle X_{n}\right\rangle /\left\langle N C \operatorname{Sym}_{n}^{+}\right\rangle
$$

Proof. For each $G\left(X_{n}\right) \in \mathbb{Q}\left\langle X_{n}\right\rangle$, we know by the previous discussion that since $\mathbb{Q}\left\langle X_{n}\right\rangle=$ $\mathrm{NCHar}_{n} \oplus\left\langle N C \operatorname{Sym}_{n}^{+}\right\rangle$, there is a unique expression $G\left(X_{n}\right)=f\left(X_{n}\right)+\sum_{A} P_{A}\left(X_{n}\right) \mathbf{m}_{A}\left[X_{n}\right]$ ( $A$ are nonsplitable) and hence we have that $G\left(X_{n}\right) \equiv f\left(X_{n}\right)\left(\bmod \left\langle N C S y m_{n}^{+}\right\rangle\right)$. Since for each non-empty set partition $A, \sigma\left(P_{A}\left(X_{n}\right) \mathbf{m}_{A}\left[X_{n}\right]\right)=\sigma\left(P_{A}\left(X_{n}\right)\right) \mathbf{m}_{A}\left[X_{n}\right] \in\left\langle N C S y m_{n}^{+}\right\rangle$ we have that $\sigma G\left(X_{n}\right) \equiv \sigma f\left(X_{n}\right)\left(\bmod \left\langle N C S y m_{n}^{+}\right\rangle\right)$. 
Theorem 8.7. As graded $\mathfrak{S}_{n}$-modules,

$$
N C \operatorname{Nar}_{n} \otimes N C \operatorname{Sym}_{n} \simeq \mathbb{Q}\left\langle X_{n}\right\rangle \text {. }
$$

Proof. Now consider the $\mathfrak{S}_{n}$ action on the space $N C H a r_{n} \otimes N C S y m_{n}$. Clearly NCSym $_{n}$ is an $\mathfrak{S}_{n}$ module since each element is $\mathfrak{S}_{n}$ invariant. For $f\left(X_{n}\right) \in N C H a r_{n}$ and for each $P\left(X_{n}\right) \in N_{C S y m}, P\left(d_{X_{n}}\right) f\left(X_{n}\right)=0$. Since $\sigma\left(P\left(d_{X_{n}}\right) f\left(X_{n}\right)\right)=\sigma\left(P\left(d_{X_{n}}\right)\right) \sigma\left(f\left(X_{n}\right)\right)=$ $P\left(d_{X_{n}}\right) \sigma\left(f\left(X_{n}\right)\right)$, hence $\sigma f\left(X_{n}\right) \in N$ Har $_{n}$ also.

Now for $G\left(X_{n}\right)=\bar{\psi}\left(\sum_{A} f_{A}\left(X_{n}\right) \otimes \mathbf{m}_{A}\left[X_{n}\right]\right)$, we notice that

$$
\sigma G\left(X_{n}\right)=\sigma\left(\sum_{A} f_{A}\left(X_{n}\right) \mathbf{m}_{A}\left[X_{n}\right]\right)=\sum_{A} \sigma\left(f_{A}\left(X_{n}\right)\right) \mathbf{m}_{A}\left[X_{n}\right]
$$

and hence $\sigma \circ \bar{\psi}=\bar{\psi} \circ(\sigma \otimes \sigma)$ and therefore the isomorphism holds on the level of $\mathfrak{S}_{n^{-}}$ modules.

We provide below a table of values of the dimensions of the graded component of degree $k$ of the space $\mathbb{Q}\left\langle X_{n}\right\rangle /\left\langle N C S y m_{n}^{+}\right\rangle$. This is computed using the table of values in section 7 and the formula given in Corollary 8.4. The rows in the table below correspond to the coefficients of $C_{n}(q)$.

\begin{tabular}{c|cccccccc}
$n / k$ & 0 & 1 & 2 & 3 & 4 & 5 & 6 & 7 \\
\hline 1 & 1 & 0 & 0 & 0 & 0 & 0 & 0 & 0 \\
2 & 1 & 1 & 1 & 1 & 1 & 1 & 1 & 1 \\
3 & 1 & 2 & 5 & 13 & 34 & 89 & 233 & 610 \\
4 & 1 & 3 & 11 & 42 & 162 & 627 & 2430 & 9423 \\
5 & 1 & 4 & 19 & 93 & 459 & 2273 & 11274 & 55964 \\
6 & 1 & 5 & 29 & 172 & 1026 & 6134 & 36712 & 219847 \\
7 & 1 & 6 & 41 & 285 & 1989 & 13901 & 97215 & 680079 \\
8 & 1 & 7 & 55 & 438 & 3498 & 27962 & 223604 & 1788406
\end{tabular}

\section{REFERENCES}

[1] M. Aguiar, F. Sottile, Structure of the Malvenuto-Reutenauer Hopf algebra of permutations, Advances in Mathematics 191 v2 (2005), 225-275.

[2] G. Bergman, P. Cohn, Symmetric elements in free powers of rings. J. London Math. Soc. 21 (1969), $525-534$.

[3] J. Berstel, C. Reutenauer, Rational series and their languages. EATCS Monographs on Theoretical Computer Science, 12. Springer-Verlag, Berlin, 1988. viii+151 pp.

[4] C. Chevalley, Invariants of finite groups generated by reflections, Amer. J. Math. 77 (1955), 778-782.

[5] L. Comtet, Sur les coefficients de l'inverse de la série formelle $\sum n ! t^{n}$. C. R. Acad. Sci. Paris Sér. A-B 275 (1972), A569-A572.

[6] F. Hivert, private communication.

[7] D. Krob and J.-Y. Thibon, Noncommutative symmetric functions IV: Quantum linear groups and Hecke algebras at $q=0$., J. Alg. Comb. 6 (1997), 339-376.

[8] A. Lascoux, private communication.

[9] I. G. Macdonald, Symmetric functions and Hall polynomials, 2nd edition, Oxford Science Publications. The Clarendon Press, Oxford University Press, New York, 1995. x+475 pp. 
[10] T. Muir, A treatise on the theory of determinants in the historical order of development. Dover Publications, New York, 1960. vii +766 pp.

[11] C. Reutenauer, Free Lie algebras. London Mathematical Society Monographs. New Series, 7. Oxford Science Publications. The Clarendon Press, Oxford University Press, New York, 1993. xviii+269 pp.

[12] M. Rosas, B. Sagan, Symmetric Functions in Noncommuting Variables. Transactions of the American Mathematical Society, to appear.

[13] B. Sagan, The symmetric group. Representations, combinatorial algorithms, and symmetric functions, 2nd edition, Graduate Text in Mathematics 203. Springer-Verlag, 2001. xvi+238 pp.

[14] N. J. A. Sloane, editor (2003), The On-Line Encyclopedia of Integer Sequences, published electronically at http://www.research.att.com/ njas/sequences/

[15] M. E. Sweedler, Hopf algebras. Mathematical Lecture Notes Series. W. A. Benjamin, 1969. vii+336 pp.

[16] R. Steinberg, Invariants of finite reflection groups, Canad. J. Math. 12 (1960), 616-618.

[17] J.-Y. Thibon, Lectures on Noncommutative Symmetric Functions, MSJ Memoirs 11, Interaction of Combinatorics and Representation Theory (2001), 39-94.

[18] M. C. Wolf, Symmetric functions of noncommutative elements, Duke Math. J. 2 (1936), 626-637.

Mathematics and Statistics, York University, 4700 Keele St., Toronto, ON, M5A 4T5, Canada

E-mail address: bergeron@mathstat.yorku.ca

E-mail address: rosas@mathstat.yorku.ca

E-mail address: zabrocki@mathstat.yorku.ca

LaCiM, Université du Québec À Montréal, Case Postale 8888, succursale Centre-ville, MontRÉAL (QuÉBEC) H3C 3P8, CANADA

E-mail address: christo@math.uqam.ca 